\title{
Mean-Square Approximation of Navier-Stokes Equations with Additive Noise in Vorticity-Velocity Formulation
}

\author{
G.N. Milstein ${ }^{1}$ and M.V. Tretyakov ${ }^{2, *}$ \\ ${ }^{1}$ Ural Federal University, Ekaterinburg, Russia \\ ${ }^{2}$ School of Mathematical Sciences, University of Nottingham, Nottingham, UK
}

Received 24 February 2020; Accepted (in revised version) 27 August 2020

\begin{abstract}
We consider a time discretization of incompressible Navier-Stokes equations with spatial periodic boundary conditions and additive noise in the vorticityvelocity formulation. The approximation is based on freezing the velocity on time subintervals resulting in a linear stochastic parabolic equation for vorticity. At each time step, the velocity is expressed via vorticity using a formula corresponding to the Biot-Savart-type law. We prove the first mean-square convergence order of the vorticity approximation.
\end{abstract}

AMS subject classifications: 65C30, 60H15, 60H35, 35Q30

Key words: Navier-Stokes equations, vorticity, numerical method, stochastic partial differential equations, mean-square convergence.

\section{Introduction}

Navier-Stokes equations (NSE), both deterministic and stochastic, are important for a number of applications and, consequently, development and analysis of numerical methods for simulation of NSE are of significant interest. The theory and applications of stochastic NSE (SNSE) can be found, e.g. in $[10,16,19]$. The literature on numerics for deterministic NSE is extensive [12,27,29] (see also references therein) while the literature on numerics for SNSE is still rather sparse, let us mention [2-6, 8, 9, 25]. In comparison with the previous works $[2-6,9,25]$ where strong approximation of SNSE in velocity formulation was considered, we here deal with the vorticity-velocity formulation, and, as far as we know, this is the first work in this direction. In [8] a similar setting to ours was used but in the context of weak approximation.

In this paper we consider two-dimensional incompressible NSE in the vorticityvelocity formulation with periodic boundary conditions and additive noise (see, e.g.

\footnotetext{
${ }^{*}$ Corresponding author. Email address: Michael. Tretyakov@nottingham.ac.uk (M.V. Tretyakov) 
[13]). In majority of papers on numerical approximation of SNSE [2-6, 9] the case of multiplicative noise is considered. The NSE with additive noise deserves a special attention due to its interesting properties $[13,16,18]$. Also, we know [23] that meansquare order of convergence of numerical methods for ordinary stochastic differential equations (SDEs) with additive noise is typically higher than with multiplicative one, which led to special consideration of SDEs with additive noise in stochastic numerics. Here we follow this path in the case of SNSE.

We propose and study time discretization of SNSE in the vorticity-velocity formulation, which is based on freezing the velocity at every time step. Consequently, at every step we just need to solve a linear parabolic stochastic PDE, which is a much simpler object than SNSE. To compute the velocity, we express it via the vorticity, i.e. via a periodic version of Biot-Savart's law (see, e.g., [15]). We prove properties, including first-order mean-square convergence, of the suggested approximation. Since we work in the vorticity-velocity formulation and aimed at reaching a higher order of meansquare convergence for the method considered, we require higher spatial smoothness of the velocity in our proofs than e.g. in [3] where mean-square convergence of fully and semi-implicit Euler schemes from [6] for SNSE with multiplicative and additive noise in the velocity formulation was considered. In the case of an additive noise, the authors of [3] proved mean-square convergence with a polynomial rate (up to $\frac{1}{4}$ ) in the time mesh. To obtain this result, they used some exponential moments bounds of the SNSE solutions analogous to the ones from [13]. Here to prove first-order meansquare convergence of our new method for SNSE in the vorticity-velocity formulation, we exploit some exponential moments bounds for vorticity.

The benefit of working within the vorticity-velocity formulation is that we do not need to deal with the divergence free condition imposed on the velocity. We remark that, as it is usual in numerical analysis, the suggested approximation can be used in practice even if the regularity conditions required for the proofs are not satisfied. In this paper our main objective is to propose a new approximation for SNSE and to prove its highest possible mean-square order of convergence under some regularity assumptions on the solution. Alternatively, one can pose the question of establishing a convergence of the proposed method under prescribed low regularity conditions, this is a topic for a possible future work (see also Remark 5.1 at the end of the paper).

The paper is organised as follows. In Section 2, after introducing the SNSE in velocity formulation, we recall function spaces required. Then we write the SNSE in the vorticity-velocity formulation (Section 2.2) and prove two auxiliary lemmas (Section 2.3) about its solution. These lemmas are of independent interest. The lemmas are later used in proving properties of the proposed approximation. We consider a one-step approximation of vorticity and its properties in Section 3. We introduce the numerical method for vorticity and prove boundedness of its moments in Section 4 . We note that to turn the proposed method into a numerical algorithm one needs to approximate a linear SPDE at every step. Various approaches can be used for this purpose as we discuss in the end of the paper, and their detailed error analysis is a subject of a future work. We prove first-order mean-square convergence of the method in Section 5. Ideas 
used in the proof of the convergence theorem are of potential interest for convergence analysis of numerical methods for a wider class of semilinear SPDEs.

\section{Preliminaries}

Let $(\Omega, \mathcal{F}, P)$ be a probability space and $\left(w(t), \mathcal{F}_{t}\right)=\left(\left(w_{1}(t), \ldots, w_{q}(t)\right)^{\top}, \mathcal{F}_{t}\right)$ be a $q$-dimensional standard Wiener process, where $\left\{\mathcal{F}_{t}, 0 \leq t \leq T\right\}$, is an increasing family of $\sigma$-subalgebras of $\mathcal{F}$ induced by $w(t)$. We consider the system of SNSE with additive noise for velocity $v$ and pressure $p$ in a viscous incompressible flow:

$$
\begin{aligned}
d v(t)= & {\left[\frac{\sigma^{2}}{2} \Delta v-(v \cdot \nabla) v-\nabla p+f(t, x)\right] d t } \\
& +\sum_{r=1}^{q} \gamma_{r}(t, x) d w_{r}(t), \quad 0<t \leq T, \quad x \in \mathbf{R}^{2},
\end{aligned}
$$

$\operatorname{div} v=0$

with spatial periodic conditions

$$
v\left(t, x+L e_{i}\right)=v(t, x), \quad p\left(t, x+L e_{i}\right)=p(t, x), \quad 0 \leq t \leq T, \quad i=1,2,
$$

and the initial condition

$$
v(0, x)=\varphi(x) .
$$

In (2.1)-(2.3), $v, f$ and $\gamma_{r}$ are two-dimensional functions; $v . \nabla=v^{1} \frac{\partial}{\partial x^{1}}+v^{2} \frac{\partial}{\partial x^{2}}$; the pressure $p$ is a scalar; $\left\{e_{i}\right\}$ is the canonical basis in $\mathbf{R}^{2}$ and $L>0$ is the period. For simplicity in writing, the periods in both directions are taken to be equal. The functions $f=f(t, x)$ and $\gamma_{r}(t, x)$ are assumed to be spatial periodic as well. Further, we require that $\gamma_{r}(t, x)$ are divergence free:

$$
\operatorname{div} \gamma_{r}(t, x)=0, \quad r=1, \ldots, q .
$$

For simplicity of proofs, we assume that the number of noises $q$ is finite but it can be shown that the theoretical results of this paper are also valid when $q$ is infinite if $\left\|\gamma_{r}(t, x)\right\|_{m}$ for some $m \geq 0$ decay exponentially fast with increase of $r$ (here $m$ is as in Assumption 2.1 below). Note that if we omit the condition (2.5) then (2.1) should be modified to include additional gradient of pressure terms $\nabla p_{r}$ for each $d w_{r}$.

\subsection{Function spaces}

We shall consider spatial periodic vector functions $u(x)=\left(u^{1}(x), u^{2}(x)\right)^{\top}: u(x+$ $\left.L e_{i}\right)=u(x), i=1,2$, where $L>0$ is the period in $i$-th direction. Denote by $Q=(0, L]^{2}$ the square of the period. We denote by $\mathbf{L}^{2}(Q)$ the Hilbert space of functions on $Q$ with the scalar product and the norm

$$
(u, v)=\int_{Q} \sum_{i=1}^{2} u^{i}(x) v^{i}(x) d x, \quad\|u\|=(u, u)^{\frac{1}{2}} .
$$


We keep the notation $|\cdot|$ for the absolute value of numbers and for the length of vectors, for example,

$$
|u(x)|=\left[\left(u^{1}(x)\right)^{2}+\left(u^{2}(x)\right)^{2}\right]^{\frac{1}{2}} .
$$

We denote by $\mathbf{H}_{p}^{m}(Q), m=0,1, \ldots$, the Sobolev space of functions which are in $\mathbf{L}^{2}(Q)$, together with all their derivatives of order less than or equal to $m$, and which are periodic functions with the period $Q$. The space $\mathbf{H}_{p}^{m}(Q)$ is a Hilbert space with the scalar product and the norm

$$
(u, v)_{m}=\int_{Q} \sum_{i=1}^{2} \sum_{\left[\alpha^{i}\right] \leq m} D^{\alpha^{i}} u^{i}(x) D^{\alpha^{i}} v^{i}(x) d x, \quad\|u\|_{m}=\left[(u, u)_{m}\right]^{\frac{1}{2}},
$$

where $\alpha^{i}=\left(\alpha_{1}^{i}, \alpha_{2}^{i}\right), \alpha_{j}^{i} \in\{0, \ldots, m\},\left[\alpha^{i}\right]=\alpha_{1}^{i}+\alpha_{2}^{i}$, and

$$
D^{\alpha^{i}}=D_{1}^{\alpha_{1}^{i}} D_{2}^{\alpha_{2}^{i}}=\frac{\partial^{\left[\alpha^{i}\right]}}{\partial\left(x^{1}\right)^{\alpha_{1}^{i}} \partial\left(x^{2}\right)^{\alpha_{2}^{i}}}, \quad i=1,2 .
$$

Note that $\mathbf{H}_{p}^{0}(Q)=\mathbf{L}^{2}(Q)$.

Introduce the Hilbert subspaces of $\mathbf{H}_{p}^{m}(Q)$ :

$$
\begin{array}{ll}
\mathbf{V}_{p}^{m}=\left\{v: v \in \mathbf{H}_{p}^{m}(Q), \operatorname{div} v=0\right\}, & m>0, \\
\mathbf{V}_{p}^{0}=\text { the closure of } \mathbf{V}_{p}^{m}, & m>0 \text { in } \mathbf{L}^{2}(Q) .
\end{array}
$$

Denote by $P$ the orthogonal projection in $\mathbf{H}_{p}^{m}(Q)$ onto $\mathbf{V}_{p}^{m}$ (we omit $m$ in the notation $P$ here). The operator $P$ is often called the Leray projection. Due to the Helmholtz-Hodge decomposition, any function $u \in \mathbf{H}_{p}^{m}(Q)$ can be represented as

$$
u=P u+\nabla g, \quad \operatorname{div} P u=0,
$$

where $g=g(x)$ is a scalar $Q$-periodic function such that $\nabla g \in \mathbf{H}_{p}^{m}(Q)$. It is natural to introduce the notation $P^{\perp} u:=\nabla g$ and hence write

$$
u=P u+P^{\perp} u
$$

with $P^{\perp} u \in\left(\mathbf{V}_{p}^{m}\right)^{\perp}=\left\{v: v \in \mathbf{H}_{p}^{m}(Q), v=\nabla g\right\}$.

Let

$$
\begin{array}{ll}
u(x)=\sum_{\mathbf{n} \in \mathbf{Z}^{2}} u_{\mathbf{n}} e^{i \frac{2 \pi}{L}(\mathbf{n}, x)}, & g(x)=\sum_{\mathbf{n} \in \mathbf{Z}^{2}} g_{\mathbf{n}} e^{i \frac{2 \pi}{L}(\mathbf{n}, x)}, \quad g_{\mathbf{0}}=0, \\
P u(x)=\sum_{\mathbf{n} \in \mathbf{Z}^{2}}(P u)_{\mathbf{n}} e^{i \frac{2 \pi}{L}(\mathbf{n}, x)}, & P^{\perp} u(x)=\nabla g(x)=\sum_{\mathbf{n} \in \mathbf{Z}^{2}}\left(P^{\perp} u\right)_{\mathbf{n}} e^{i \frac{2 \pi}{L}(\mathbf{n}, x)}
\end{array}
$$

be the Fourier expansions of $u, g, P u$, and $P^{\perp} u=\nabla g$. Here $u_{\mathbf{n}},(P u)_{\mathbf{n}}$, and $\left(P^{\perp} u\right)_{\mathbf{n}}=$ $(\nabla g)_{\mathbf{n}}$ are two-dimensional vectors and $g_{\mathbf{n}}$ are scalars. We note that $g_{\mathbf{0}}$ can be any 
real number but for definiteness we set $g_{0}=0$ without loss of generality [11]. The coefficients $(P u)_{\mathbf{n}},\left(P^{\perp} u\right)_{\mathbf{n}}$, and $g_{\mathbf{n}}$ can be easily expressed in terms of $u_{\mathbf{n}}$ :

$$
\begin{aligned}
& (P u)_{\mathbf{n}}=u_{\mathbf{n}}-\frac{u_{\mathbf{n}}^{\top} \mathbf{n}}{|\mathbf{n}|^{2}} \mathbf{n}, \quad\left(P^{\perp} u\right)_{\mathbf{n}}=i \frac{2 \pi}{L} g_{\mathbf{n}} \mathbf{n}=\frac{u_{\mathbf{n}}^{\top} \mathbf{n}}{|\mathbf{n}|^{2}} \mathbf{n}, \\
& g_{\mathbf{n}}=-i \frac{L}{2 \pi} \frac{u_{\mathbf{n}}^{\top} \mathbf{n}}{|\mathbf{n}|^{2}}, \quad \mathbf{n} \neq \mathbf{0}, \quad g_{\mathbf{0}}=0 .
\end{aligned}
$$

We have

$$
\nabla e^{i \frac{2 \pi}{L}(\mathbf{n}, x)}=\mathbf{n} e^{i \frac{2 \pi}{L}(\mathbf{n}, x)} \cdot i \frac{2 \pi}{L},
$$

hence $u_{\mathbf{n}} e^{i \frac{2 \pi}{L}(\mathbf{n}, x)} \in \mathbf{V}_{p}^{m}$ if and only if $\left(u_{\mathbf{n}}, \mathbf{n}\right)=0$. We obtain from here that the orthogonal basis of the subspace $\left(\mathbf{V}_{p}^{m}\right)^{\perp}$ consists of $\mathbf{n} e^{i \frac{2 \pi}{L}(\mathbf{n}, x)}, \mathbf{n} \in \mathbf{Z}^{2}, \mathbf{n} \neq \mathbf{0}$; and the orthogonal basis of $\mathbf{V}_{p}^{m}$ consists of vectors (for $\mathbf{n} \neq 0$ ):

$$
\left[\begin{array}{r}
-n_{2} \\
n_{1}
\end{array}\right] e^{i \frac{2 \pi}{L}(\mathbf{n}, x)}, \quad \mathbf{n}=\left(n_{1}, n_{2}\right)^{\top},
$$

which are orthogonal to $\mathbf{n}$.

In the rest of the paper all functions $u \in \mathbf{H}_{p}^{m}(Q)$ will be assumed to have zero space average (see e.g. [11]), i.e.

$$
\int_{Q} u(x)=0 .
$$

In this case the Fourier series expansion for $u(x)$ does not contain the constant term and $\sum_{\mathbf{n} \in \mathbf{Z}^{2}}$ in (2.7) can be replaced by $\sum_{\mathbf{n} \in \mathbf{Z}^{2}, \mathbf{n} \neq 0}$, which in what follows we will write as simply $\sum$.

We recall Parseval's identity

$$
\|u\|^{2}=\int_{Q}|u(x)|^{2} d x=L^{2} \sum\left|u_{\mathbf{n}}\right|^{2} .
$$

We also note the following two relationships. Since the vector field $u=u(x)$ is real valued, we have

$$
u_{-\mathbf{n}}=\bar{u}_{\mathbf{n}}, \quad \mathbf{n} \in \mathbf{Z}^{2}, \quad \mathbf{n} \neq 0,
$$

where $\bar{u}_{\mathbf{n}}$ denotes the complex conjugate of $u_{\mathbf{n}}$. The divergence-free condition reads

$$
u_{\mathbf{n}}^{\top} \mathbf{n}=\left(u_{\mathbf{n}}, \mathbf{n}\right)=\sum_{k=1}^{2} u_{\mathbf{n}}^{k} \mathbf{n}^{k}=0 .
$$

We will need the following estimate for the tri-linear form (see [7, p. 50, Eq. (6.10)] or [28, p. 12, Eq. (2.29)]):

$$
|((v . \nabla) u, g)| \leq K\|v\|_{m_{1}}\|u\|_{m_{2}+1}\|g\|_{m_{3}}
$$


where $K>0$ is a constant, $m_{1}, m_{2}$ and $m_{3}$ are such that $m_{1}+m_{2}+m_{3} \geq 1$ and $\left(m_{1}, m_{2}, m_{3}\right) \neq(0,0,1),(0,1,0),(1,0,0)$, and $u, v, g$ are arbitrary functions from the corresponding spaces. Further, we recall the standard interpolation inequality for Sobolev spaces (see e.g. [28, p. 11]):

$$
\|u\|_{m} \leq\|u\|_{m_{1}}^{1-l}\|u\|_{m_{2}}^{l}
$$

where $m=(1-l) m_{1}+l m_{2}, m_{1}, m_{2} \geq 0, l \in(0,1)$, and $u \in \mathbf{H}_{p}^{\max \left(m_{1}, m_{2}\right)}(Q)$. For any $c>0$, we get from (2.13) and Young's inequality:

$$
\|u\|_{m}^{2} \leq\|u\|_{m_{1}}^{2-2 l}\|u\|_{m_{2}}^{2 l} \leq(1-l) c\|u\|_{m_{1}}^{2}+l c^{1-\frac{1}{l}}\|u\|_{m_{2}}^{2} .
$$

Let us take $m_{1}=1, m_{2}=0$ and $m_{3}=\frac{1}{2}$ in (2.12), then we get (see also [13, p. 1028, Eq. (A6)]) for any $c_{1}>0$ and $c_{2}>0$ :

$$
\begin{aligned}
|((v . \nabla) u, g)| & \leq K\|v\|_{1}\|u\|_{1}\|g\|_{\frac{1}{2}} \leq \frac{K^{2}}{4 c_{1}}\|g\|_{\frac{1}{2}}^{2}+c_{1}\|v\|_{1}^{2}\|u\|_{1}^{2} \\
& \leq \frac{K^{2}}{4 c_{1}}\|g\|_{\frac{1}{2}}^{2}+c_{1}\|v\|_{1}^{2}\|u\|_{1}^{2} \\
& \leq c_{2}\|g\|_{1}^{2}+\frac{K^{4}}{64 c_{1}^{2} c_{2}}\|g\|^{2}+c_{1}\|v\|_{1}^{2}\|u\|_{1}^{2},
\end{aligned}
$$

where we used (2.14) with $m=\frac{1}{2}, m_{1}=1, m_{2}=0$ and $l=\frac{1}{2}$ (fractional $\mathbf{H}_{p}^{m}(Q)$ spaces are defined in the usual way via the Fourier series expansions, see e.g. [28, pp. 7-8]).

We also recall (see e.g. [11, p. 20 Eq. (4.14)]) Poincare's inequality for functions $u \in \mathbf{H}_{p}^{1}(Q)$ satisfying (2.10):

$$
\|u\| \leq \alpha\|\nabla u\|
$$

for some constant $\alpha>0$ which depends only on the period $L$. We note that here and in what follows: when $u(x)$ is a vector, $\nabla u(x)$ means the matrix with elements $\frac{\partial u^{i}}{\partial x^{j}}$ and $\|\nabla u\|$ means $L^{2}$-norm of the Frobenius norm of the matrix $\nabla u(x)$.

We make the following assumption for the NSE problem (2.1)-(2.4).

Assumption 2.1. We assume that the coefficients $f(t, x)$ and $\gamma_{r}(s, x), r=1, \ldots, q$, belong to $\mathbf{H}_{p}^{m+1}(Q)$ and the initial condition $\varphi(x)$ belongs to $\mathbf{H}_{p}^{m+2}(Q)$ for some $m \geq 0$.

Under this assumption the problem (2.1)-(2.4) has a unique solution $v(t, x), p(t, x)$, $(t, x) \in[0, T] \times \mathbf{R}^{2}$, so that for $l \geq 2[16,17]$ :

$$
E\|v(t, \cdot)\|_{m+2}^{l} \leq K,
$$

where $K>0$ may depend on $l, m, T, f(t, x), \gamma_{r}(t, x)$, and $\varphi(x)$. The solution $v(t, x)$, $p(t, x),(t, x) \in[0, T] \times \mathbf{R}^{2}$, to (2.1)-(2.4) is $\mathcal{F}_{t}$-adaptive, $v(t, \cdot) \in \mathbf{V}_{p}^{m+2}$ and $\nabla p(t, \cdot) \in$ $\left(\mathbf{V}_{p}^{m+2}\right)^{\perp}$ for every $t \in[0, T]$ and $\boldsymbol{\omega} \in \Omega$. We note that if we were interested in variational solutions of (2.1)-(2.4) then it is more natural to put $m \geq-1$ in Assumption 2.1, but here our focus is on the vorticity formulation and then it is natural to require more, $m \geq 0$. Consequently, we are dealing with the setting in which the solution of (2.1)(2.4) is analytically (i.e., PDE-wise) strong. 


\subsection{Equation for vorticity}

Introduce the vorticity $\omega$ :

$$
\omega(x)=\operatorname{curl} v=\frac{\partial v^{2}}{\partial x^{1}}(x)-\frac{\partial v^{1}}{\partial x^{2}}(x) .
$$

Note that $\omega(x)$ is a scalar. Analogously to (2.7), we write the Fourier expansion for $\omega$ :

$$
\omega(x)=\sum \omega_{\mathbf{n}} e^{i \frac{2 \pi}{L}\left(\mathbf{n}^{1} x^{1}+\mathbf{n}^{2} x^{2}\right)},
$$

where

$$
\omega_{\mathbf{n}}=i \frac{2 \pi}{L}\left(v_{\mathbf{n}}^{2} \mathbf{n}^{1}-v_{\mathbf{n}}^{1} \mathbf{n}^{2}\right) .
$$

It is known (see, e.g. [15, p. 50]) that $v$ is expressed through $\omega$ as

$$
v(x)=\frac{L i}{2 \pi}\left[\begin{array}{c}
\sum \frac{1}{|\mathbf{n}|^{2}} e^{i \frac{2 \pi}{L}(\mathbf{n}, x)} \omega_{\mathbf{n}} \mathbf{n}^{2} \\
-\sum \frac{1}{|\mathbf{n}|^{2}} e^{i \frac{2 \pi}{L}(\mathbf{n}, x)} \omega_{\mathbf{n}} \mathbf{n}^{1}
\end{array}\right]:=U \omega .
$$

Taking the curl of (2.1) gives the evolution equation for the vorticity $\omega=\operatorname{curl} v$ :

$$
d \omega=\left[\frac{\sigma^{2}}{2} \Delta \omega-(v \cdot \nabla) \omega+g(t, x)\right] d t+\sum_{r=1}^{q} \mu_{r}(t, x) d w_{r}(t),
$$

where $g=\operatorname{curl} f$ and $\mu_{r}=\operatorname{curl} \gamma_{r}$. The vorticity satisfies the initial and periodic boundary conditions

$$
\omega(0, x)=\operatorname{curl} \varphi(x):=\phi(x)
$$

and spatial periodic conditions

$$
\omega\left(t, x+L e_{i}\right)=\omega(t, x), \quad i=1,2, \quad 0 \leq t \leq T .
$$

Using the linear operator $U$ from (2.18), we can re-write (2.19) as (see e.g. [13]):

$$
d \omega=\left[\frac{\sigma^{2}}{2} \Delta \omega-(U \omega \cdot \nabla) \omega+g(t, x)\right] d t+\sum_{r=1}^{q} \mu_{r}(t, x) d w_{r}(t) .
$$

Similarly to the solution $v(t, x)$ of (2.1)-(2.4), the solution $\omega(t, x)$ to the vorticity problem (2.19)-(2.21) under Assumption 2.1 is so that for $p \geq 2$ :

$$
E\|\omega(t, \cdot)\|_{m+1}^{p} \leq K
$$

where $K>0$ depends on $p, m, g, \mu_{r}$ and $\phi$. Note that under Assumption 2.1 the coefficients $g(t, x)$ and $\mu_{r}(s, x), r=1, \ldots, q$, belong to $\mathbf{H}_{p}^{m}(Q)$ and the initial condition $\phi(x)$ 
belongs to $\mathbf{H}_{p}^{m+1}(Q)$. As it is clear from the context, we are dealing here with solutions understood in the strong sense probabilistically.

In future we will need the following estimates. One can obtain from (2.18) that for $m \geq 1$

$$
\|v\|_{m}=\|U \omega\|_{m} \leq K\|\omega\|_{m-1}
$$

for some $K>0$. Further, we note that

$$
\|\omega\|_{1}^{2}=\|\omega\|^{2}+\|\nabla \omega\|^{2}
$$

and then by (2.16)

$$
\|\omega\|_{1}^{2} \leq K\|\nabla \omega\|^{2}
$$

for some $K>0$. Using (2.15) and (2.24)-(2.26), we get for $\omega, v, g$ from appropriate spaces and arbitrary $c_{1}>0$ and $c_{2}>0$ :

$$
\begin{aligned}
|((U \omega \cdot \nabla) v, g)| & \leq c_{2}\|g\|_{1}^{2}+\frac{K^{4}}{64 c_{1}^{2} c_{2}}\|g\|^{2}+c_{1}\|U \omega\|_{1}^{2}\|v\|_{1}^{2} \\
& \leq c_{2}\|\nabla g\|^{2}+\left(c_{2}+\frac{K^{4}}{64 c_{1}^{2} c_{2}}\right)\|g\|^{2}+K c_{1}\|\omega\|^{2}\|\nabla v\|^{2} \\
& =c_{2}\|\nabla g\|^{2}+K\|g\|^{2}+c_{3}\|\omega\|^{2}\|\nabla v\|^{2},
\end{aligned}
$$

where in the third line $c_{3}>0$ is an arbitrary constant and $K>0$ is some constant dependent on $c_{2}$ and $c_{3}$ (it differs from $K>0$ in the first and second lines but this should not cause any confusion).

\subsection{Two technical lemmas}

For proving convergence of the numerical method in Section 5, we need two further properties of the solution $\omega(t, x)$ to the SNSE (2.19)-(2.21) formulated in the next two lemmas, which are of independent interest.

It is convenient to introduce the notation for the solution $\omega(t, x)$ of the problem (2.19)-(2.21), which reflects its dependence on the initial condition $\phi(x)$ prescribed at time $s \leq t$ :

$$
\omega(t, x)=\omega(t, x ; s, \phi) .
$$

Let us prove a technical lemma on exponential moments bounds for vorticity, which is related to [13, Lemmas 4.10(1) and A.1].

Lemma 2.1. Let Assumption 2.1 hold with $m=0$. There exist constants $\beta_{0}>0$ and $\alpha>0$ such that for any $\beta \in\left(0, \beta_{0}\right]$ and $0 \leq t \leq t+h \leq T$ :

$$
\begin{aligned}
& E \exp \left(\beta\left[\|\omega(t+h, \cdot ; t, \phi)\|^{2}-\|\phi\|^{2}\right]+\beta \frac{\sigma^{2}}{4} \int_{t}^{t+h}\|\nabla \omega(s, \cdot ; t, \phi)\|^{2} d s\right) \\
\leq & \exp \left(\beta \int_{t}^{t+h}\left(\frac{2}{\alpha \sigma^{2}}\|g(s, \cdot)\|^{2}+\sum_{r=1}^{q}\left\|\mu_{r}(s, \cdot)\right\|^{2}\right) d s\right) .
\end{aligned}
$$


Proof. By the Ito formula, integration by parts and using $\operatorname{div} v(t, x)=0$, we obtain

$$
\begin{aligned}
\frac{1}{2} d\|\omega(s, \cdot)\|^{2}= & {\left[-\frac{\sigma^{2}}{2}\|\nabla \omega(s, \cdot)\|^{2}+(g(s, \cdot), \omega(s, \cdot))+\frac{1}{2} \sum_{r=1}^{q}\left\|\mu_{r}(s, \cdot)\right\|^{2}\right] d s } \\
& +\sum_{r=1}^{q}\left(\mu_{r}(s, \cdot), \omega(s, \cdot)\right) d w_{r}(s), \quad t<s \leq t+h,
\end{aligned}
$$

$\|\omega(t, \cdot)\|^{2}=\|\phi\|^{2}$.

Using the elementary inequality, we get for any $\alpha>0$ :

$$
\begin{aligned}
& \frac{1}{2} d\|\omega(s, \cdot)\|^{2} \\
\leq & {\left[-\frac{\sigma^{2}}{2}\|\nabla \omega(s, \cdot)\|^{2}+\frac{1}{\alpha \sigma^{2}}\|g(s, \cdot)\|^{2}+\frac{\sigma^{2}}{4} \alpha\|\omega(s, \cdot)\|^{2}+\frac{1}{2} \sum_{r=1}^{q}\left\|\mu_{r}(s, \cdot)\right\|^{2}\right] d s } \\
& +\sum_{r=1}^{q}\left(\mu_{r}(s, \cdot), \omega(s, \cdot)\right) d w_{r}(s) .
\end{aligned}
$$

By Poincare's inequality (2.16), for some $\alpha>0$, we have

$$
\|\nabla \omega(t, \cdot)\|^{2} \geq \alpha\|\omega(t, \cdot)\|^{2} .
$$

By (2.31), we obtain

$$
\begin{aligned}
d\|\omega(s, \cdot)\|^{2} \leq & {\left[-\frac{\sigma^{2}}{4}\|\nabla \omega(s, \cdot)\|^{2}-\frac{\sigma^{2}}{4} \alpha\|\omega(s, \cdot)\|^{2}+\frac{2}{\alpha \sigma^{2}}\|g(s, \cdot)\|^{2}+\sum_{r=1}^{q}\left\|\mu_{r}(s, \cdot)\right\|^{2}\right] d s } \\
& +2 \sum_{r=1}^{q}\left(\mu_{r}(s, \cdot), \omega(s, \cdot)\right) d w_{r}(s)
\end{aligned}
$$

then for any $c>0$

$$
\begin{aligned}
& c\|\omega(t+h, \cdot)\|^{2}-c\|\phi\|^{2}+c \frac{\sigma^{2}}{4} \int_{t}^{t+h}\|\nabla \omega(s, \cdot)\|^{2} d s \\
& -c \int_{t}^{t+h}\left(\frac{2}{\alpha \sigma^{2}}\|g(s, \cdot)\|^{2}+\sum_{r=1}^{q}\left\|\mu_{r}(s, \cdot)\right\|^{2}\right) d s \\
& \leq 2 c \int_{t}^{t+h} \sum_{r=1}^{q}\left(\mu_{r}(s, \cdot), \omega(s, \cdot)\right) d w_{r}(s)-\alpha \frac{\sigma^{2}}{4} c \int_{t}^{t+h}\|\omega(s, \cdot)\|^{2} d s .
\end{aligned}
$$

Let

$$
M\left(t, t^{\prime}\right):=2 c \int_{t}^{t^{\prime}} \sum_{r=1}^{q}\left(\mu_{r}(s, \cdot), \omega(s, \cdot)\right) d w_{r}(s),
$$


which is a continuous $L^{2}$-martingale with the quadratic variation

$$
<M>\left(t, t^{\prime}\right):=4 c^{2} \int_{t}^{t^{\prime}} \sum_{r=1}^{q}\left(\mu_{r}(s, \cdot), \omega(s, \cdot)\right)^{2} d s .
$$

There exists a constant $\beta_{0}>0$ (independent of $h$ and $c$ ) so that for all $\beta \in\left(0, \beta_{0}\right]$ :

$$
\alpha \frac{\sigma^{2}}{4} c \int_{t}^{t^{\prime}}\|\omega(s, \cdot)\|^{2} d s \geq \frac{\beta}{2 c}<M>\left(t, t^{\prime}\right) .
$$

Hence

$$
\begin{aligned}
& c\|\omega(t+h, \cdot)\|^{2}-c\|\phi\|^{2}+c \frac{\sigma^{2}}{4} \int_{t}^{t+h}\|\nabla \omega(s, \cdot)\|^{2} d s \\
& -c \int_{t}^{t+h}\left(\frac{2}{\alpha \sigma^{2}}\|g(s, \cdot)\|^{2}+\sum_{r=1}^{q}\left\|\mu_{r}(s, \cdot)\right\|^{2}\right) d s \\
\leq & M(t, t+h)-\frac{\beta}{2 c}<M>(t, t+h) .
\end{aligned}
$$

For $c=\beta$, the right-hand side of (2.34) is logarithm of a local exponential martingale and therefore

$$
\begin{array}{r}
E \exp \left[\beta\left\|\omega\left(t+h \wedge \tau_{n}, \cdot\right)\right\|^{2}-\beta\|\phi\|^{2}+\beta \frac{\sigma^{2}}{4} \int_{t}^{t+h \wedge \tau_{n}}\|\nabla \omega(s, \cdot)\|^{2} d s\right. \\
\left.-\beta \int_{t}^{t+h \wedge \tau_{n}}\left(\frac{2}{\alpha \sigma^{2}}\|g(s, \cdot)\|^{2}+\sum_{r=1}^{q}\left\|\mu_{r}(s, \cdot)\right\|^{2}\right) d s\right] \leq 1
\end{array}
$$

where $\tau_{n}=\inf \{s>t:<M>(t, s) \geq n\}$ for a natural number $n$. Tending $n$ to infinity, we arrive at (2.28). Lemma 2.1 is proved.

It follows from (2.28) that

$$
\begin{aligned}
& E \exp \left(\beta \frac{\sigma^{2}}{4} \int_{t}^{t+h}\|\nabla \omega(s, \cdot ; t, \phi)\|^{2} d s\right) \\
\leq & \exp \left(\beta\|\phi\|^{2}+\beta \int_{t}^{t+h}\left(\frac{2}{\alpha \sigma^{2}}\|g(s, \cdot)\|^{2}+\sum_{r=1}^{q}\left\|\mu_{r}(s, \cdot)\right\|^{2}\right) d s\right) .
\end{aligned}
$$

We also pay attention that the proof of Lemma 2.1 is not relying on smallness of the time step $h$, and after replacing $t$ with 0 and $t+h$ with $T$ the result remains valid:

$$
\begin{aligned}
& E \exp \left(\beta \frac{\sigma^{2}}{4} \int_{0}^{T}\|\nabla \omega(s, \cdot ; 0, \phi)\|^{2} d s\right) \\
\leq & \exp \left(\beta\|\phi\|^{2}+\beta \int_{0}^{T}\left(\frac{2}{\alpha \sigma^{2}}\|g(s, \cdot)\|^{2}+\sum_{r=1}^{q}\left\|\mu_{r}(s, \cdot)\right\|^{2}\right) d s\right) .
\end{aligned}
$$

We now prove the next lemma which gives us dependence of the solution $\omega(s, x ; t, \phi)$ on the initial data. 
Lemma 2.2. Let Assumption 2.1 hold with $m=3$ and $\phi_{i}(t, x), i=1,2$, be $\mathcal{F}_{t}$-measurable processes satisfying (2.23) with $m=3$. There exists a constant $c_{0}>0$ such that for every $c \in\left(0, c_{0}\right)$ there is a sufficiently small $h>0$ so that we have for $t \leq s \leq t+h$ :

$$
\omega\left(s, x ; t, \phi_{1}(t, \cdot)\right)-\omega\left(s, x ; t, \phi_{2}(t, \cdot)\right)=\phi_{1}(t, x)-\phi_{2}(t, x)+\eta(s, x),
$$

for which

$$
\begin{aligned}
& \left\|\omega\left(s, \cdot ; t, \phi_{1}\right)-\omega\left(s, \cdot ; t, \phi_{2}\right)\right\|^{2} \\
\leq & \left\|\phi_{1}(t, \cdot)-\phi_{2}(t, \cdot)\right\|^{2} \exp \left(K(s-t)+c \int_{t}^{s}\left\|\nabla \omega\left(s^{\prime}, \cdot ; t, \phi_{1}(t, \cdot)\right)\right\|^{2} d s^{\prime}\right),
\end{aligned}
$$

where $K>0$ is a constant.

The process $\eta(s)$ satisfies the following estimate

$$
\|\eta(s, \cdot)\|^{2} \leq(s-t)\left\|\phi_{1}(t, \cdot)-\phi_{2}(t, \cdot)\right\|^{2}+C(s, \omega)(s-t)^{3},
$$

where $C(s, \omega)>0$ is an $\mathcal{F}_{s}$-adapted process with bounded moments of a sufficiently high order.

Proof. Let

$$
\theta(s, x):=\omega\left(s, x ; t, \phi_{1}\right)-\omega\left(s, x ; t, \phi_{2}\right) .
$$

We have

$$
\begin{aligned}
& d \theta(s, x)=\left[\frac{\sigma^{2}}{2} \Delta \theta-(U \theta . \nabla) \omega\left(s, \cdot ; t, \phi_{1}\right)-\left(U \omega\left(s, \cdot ; t, \phi_{2}\right) . \nabla\right) \theta\right] d s, \quad t<s \leq t+h, \\
& \theta(t, x)=\phi_{1}(t, x)-\phi_{2}(t, x) .
\end{aligned}
$$

Then

$$
\begin{aligned}
& \frac{1}{2} d\|\theta(s, \cdot)\|^{2} \\
= & {\left[-\frac{\sigma^{2}}{2}\|\nabla \theta(s, \cdot)\|^{2}-\left((U \theta(s, \cdot) \cdot \nabla) \omega\left(s, \cdot ; t, \phi_{1}\right), \theta(s, \cdot)\right)\right] d s, \quad t<s \leq t+h, } \\
& \|\theta(t, \cdot)\|^{2}=\left\|\phi_{1}(t, \cdot)-\phi_{2}(t, \cdot)\right\|^{2} .
\end{aligned}
$$

Using the inequality (2.27) with $c_{2}=\frac{\sigma^{2}}{4}$, we obtain that there exists $K>0$ such that for any $c>0$

$$
\begin{aligned}
& 2\left|\left((U \theta(s, \cdot) \cdot \nabla) \omega\left(s, \cdot ; t, \phi_{1}\right), \theta(s, \cdot)\right)\right| \\
\leq & \frac{\sigma^{2}}{2}\|\nabla \theta(s, \cdot)\|^{2}+K\|\theta(s, \cdot)\|^{2}+c\left\|\nabla \omega\left(s, \cdot ; t, \phi_{1}\right)\right\|^{2}\|\theta(s, \cdot)\|^{2}
\end{aligned}
$$

and hence

$$
d\|\theta(s, \cdot)\|^{2} \leq\left[K+c\left\|\nabla \omega\left(s, \cdot ; t, \phi_{1}\right)\right\|^{2}\right]\|\theta(s, \cdot)\|^{2} d s, \quad t<s \leq t+h,
$$


which implies

$$
\begin{aligned}
& \|\theta(s, \cdot)\|^{2} \\
\leq & \left\|\phi_{1}(t, \cdot)-\phi_{2}(t, \cdot)\right\|^{2} \exp \left(K(s-t)+c \int_{t}^{s}\left\|\nabla \omega\left(s^{\prime}, \cdot ; t, \phi_{1}(t, \cdot)\right)\right\|^{2} d s^{\prime}\right) .
\end{aligned}
$$

Thus we have proved the inequality (2.38).

Let us now prove (2.39). We have

$$
\begin{aligned}
& \eta(s, x) \\
= & \int_{t}^{s}\left[\frac{\sigma^{2}}{2} \Delta \theta-(U \theta . \nabla) \omega\left(s^{\prime}, x ; t, \phi_{1}\right)-\left(U \omega\left(s^{\prime}, x ; t, \phi_{2}\right) . \nabla\right) \theta\right] d s^{\prime}, \quad t<s \leq t+h,
\end{aligned}
$$

which together with (2.17) and (2.23) implies that

$$
\|\eta(s, x)\| \leq C(s, \omega)(s-t),
$$

where $C(s, \omega)>0$ is an $\mathcal{F}_{s}$-adapted process with bounded moments of a sufficiently high order. Differentiate (2.43)

$$
\begin{aligned}
& \frac{\partial}{\partial x^{i}} \eta(s, x) \\
= & \int_{t}^{s}\left[\frac{\sigma^{2}}{2} \Delta \frac{\partial}{\partial x^{i}} \theta-\frac{\partial}{\partial x^{i}}(U \theta \cdot \nabla) \omega\left(s^{\prime}, x ; t, \phi_{1}\right)-\frac{\partial}{\partial x^{i}}\left(U \omega\left(s^{\prime}, x ; t, \phi_{2}\right) . \nabla\right) \theta\right] d s^{\prime},
\end{aligned}
$$

and observe that, again due to (2.17) and (2.23), the inequality (2.44) is also valid for $\|\nabla \eta(s, x)\|$ :

$$
\|\nabla \eta(s, x)\| \leq C(s, \omega)(s-t) .
$$

By further differentiating (2.45) and using (2.17) and (2.23), we can get

$$
\|\Delta \eta(s, x)\| \leq C(s, \omega)(s-t) .
$$

Note that we need $m=3$ in the conditions of the lemma to obtain (2.47).

We have

$$
\begin{aligned}
& d\left\|\eta\left(s^{\prime}, x\right)\right\|^{2} \\
= & {\left[\left(\sigma^{2} \Delta \theta, \eta\right)-2\left((U \theta \cdot \nabla) \omega\left(s^{\prime}, \cdot ; t, \phi_{1}\right), \eta\right)-2\left(\left(U \omega\left(s^{\prime}, \cdot ; t, \phi_{2}\right) \cdot \nabla\right) \theta, \eta\right)\right] d s^{\prime} } \\
\leq & {\left[-\left(\sigma^{2} \nabla\left(\phi_{1}(t, \cdot)-\phi_{2}(t, \cdot)\right), \nabla \eta\right)-2\left((U \theta \cdot \nabla) \omega\left(s^{\prime}, \cdot ; t, \phi_{1}\right), \eta\right)\right.} \\
& \left.-2\left(\left(U \omega\left(s^{\prime}, \cdot ; t, \phi_{2}\right) \cdot \nabla\right) \theta, \eta\right)\right] d s^{\prime} .
\end{aligned}
$$

Using integration by parts, (2.38) and (2.47), we get

$$
\begin{aligned}
& \left|\left(\sigma^{2} \nabla\left(\phi_{1}(t, \cdot)-\phi_{2}(t, \cdot)\right), \nabla \eta\right)\right| \\
= & \sigma^{2}\left|\left(\phi_{1}(t, \cdot)-\phi_{2}(t, \cdot), \Delta \eta\right)\right| \leq C\left(s^{\prime}, \omega\right)\left(s^{\prime}-t\right)\left\|\phi_{1}(t, \cdot)-\phi_{2}(t, \cdot)\right\| .
\end{aligned}
$$


By (2.12) with $m_{1}=1, m_{2}=0$, and $m_{3}=1$, and using (2.24), (2.23), (2.38), (2.44) and (2.46), we obtain

$$
\begin{aligned}
\left|2\left((U \theta . \nabla) \omega\left(s, \cdot ; t, \phi_{1}\right), \eta\right)\right| & \leq K\|U \theta\|_{1}\|\omega\|_{1}\|\eta\|_{1} \leq K\|\theta\|\|\omega\|_{1}\|\eta\|_{1} \\
& \leq C\left(s^{\prime}, \omega\right)\left(s^{\prime}-t\right)\left\|\phi_{1}(t, \cdot)-\phi_{2}(t, \cdot)\right\| .
\end{aligned}
$$

And by (2.12) with $m_{1}=2, m_{2}=0$, and $m_{3}=0$, and using (2.24), (2.23), (2.38), (2.44) and (2.46), we arrive at

$$
\begin{aligned}
\left|2\left(\left(U \omega\left(s^{\prime}, \cdot ; t, \phi_{2}\right) . \nabla\right) \theta, \eta\right)\right| & =2\left|\left(\left(U \omega\left(s^{\prime}, \cdot ; t, \phi_{2}\right) . \nabla\right) \eta, \theta\right)\right| \leq K\|\omega\|_{1}\|\eta\|_{1}\|\theta\| \\
& \leq C\left(s^{\prime}, \omega\right)\left(s^{\prime}-t\right)\left\|\phi_{1}(t, \cdot)-\phi_{2}(t, \cdot)\right\| .
\end{aligned}
$$

Then we have

$$
\begin{aligned}
d\left\|\eta\left(s^{\prime}, x\right)\right\|^{2} & \leq C\left(s^{\prime}, \omega\right)\left(s^{\prime}-t\right)\left\|\phi_{1}(t, \cdot)-\phi_{2}(t, \cdot)\right\| d s^{\prime} \\
& \leq\left\|\phi_{1}(t, \cdot)-\phi_{2}(t, \cdot)\right\|^{2} d s^{\prime}+\frac{1}{4} C^{2}\left(s^{\prime}, \omega\right)\left(s^{\prime}-t\right)^{2} d s^{\prime},
\end{aligned}
$$

from which (2.39) follows. Lemma 2.2 is proved.

\section{One-step approximation}

Consider the uniform partition $0=t_{0}<\cdots<t_{N}=T$ of the interval $[0, T]$ and the time step $h=\frac{T}{N}$ (we restrict ourselves to the uniform time discretization for simplicity only). In this section we derive a one-step approximation for the vorticity and study its properties.

We can approximate SNSE (2.19)-(2.21) by freezing the velocity:

$$
v(t, x) \approx \hat{v}(t, x):=v\left(t_{k}, x\right):=\hat{v}(x), \quad t_{k}<t \leq t_{k+1},
$$

and obtain an approximation $\tilde{\omega}(t, x)$ of $\omega(t, x)$ on $t_{k} \leq t \leq t_{k+1}$, as follows

$$
\begin{array}{ll}
d \tilde{\omega}=\left[\frac{\sigma^{2}}{2} \Delta \tilde{\omega}-(\hat{v} . \nabla) \tilde{\omega}+g(t, x)\right] d t+\sum_{r=1}^{q} \mu_{r}(t, x) d w_{r}(t), & t_{k}<t \leq t_{k+1}, \\
\tilde{\omega}\left(t_{k}, x\right)=\omega\left(t_{k}, x\right), \quad \tilde{\omega}\left(t_{k}, x+L e_{j}\right)=\tilde{\omega}\left(t_{k}, x\right), & j=1,2 .
\end{array}
$$

It is not difficult to see that the local error $\delta_{\omega}(t, x)=\tilde{\omega}(t, x)-\omega(t, x), t_{k} \leq t \leq t_{k+1}$, for the approximation $\tilde{\omega}(t, x)$ of the solution $\omega(t, x)$ of SNSE (2.19)-(2.21) satisfies the problem

$$
\begin{aligned}
& d \delta_{\omega}=\left[\frac{\sigma^{2}}{2} \Delta \delta_{\omega}-(v \cdot \nabla) \delta_{\omega}-((v-\hat{v}) \cdot \nabla) \tilde{\omega}\right] d t \\
& \delta_{\omega}\left(t_{k}, x\right)=0 .
\end{aligned}
$$


Moments of $\|\tilde{\omega}\|_{3}$ (and hence of $\left\|\delta_{\omega}\right\|_{3}$ ) up to a sufficiently high order are bounded under Assumption 2.1 with $m=2$ : for $t_{k}<t \leq t_{k+1}$ and $p \geq 1$ :

$$
E\|\tilde{\omega}(t, \cdot)\|_{3}^{2 p} \leq K
$$

where $K>0$ is a constant. This boundedness (3.6) can be proved by arguments similar to boundedness of moments of the global approximation (see Theorems 4.1 and 4.2).

To obtain estimates for the one-step error $\delta_{\omega}$, we first prove the following lemma.

Lemma 3.1. Let Assumption 2.1 hold with $m=1$. For $v(t, x)$ from $(2.1)-(2.4), \hat{v}(x)$ from (3.1), and $\tilde{\omega}(t, x)$ from (3.2)-(3.3), we have for $t_{k}<t \leq t_{k+1}$ and sufficiently small $h>0$ :

$$
\begin{aligned}
& \left\|E\left[((v-\hat{v}) . \nabla) \tilde{\omega} \mid \mathcal{F}_{t_{k}}\right]\right\| \leq C\left(t_{k}, \omega\right) h, \\
& \left(E\|v-\hat{v}\|^{2 p}\right)^{\frac{1}{2 p}} \leq K h^{\frac{1}{2}}, \quad p \geq 1,
\end{aligned}
$$

where $C\left(t_{k}, \omega\right)>0$ is an $\mathcal{F}_{t_{k}}$-measurable random variable with moments of a sufficiently high order bounded by a constant independent of $h$ and $K>0$ is a constant independent of $h$.

Proof. From (2.1) and (3.1), we have for $t_{k}<t \leq t_{k+1}$ :

$$
\begin{aligned}
& v(t, x)-\hat{v}(x) \\
= & \int_{t_{k}}^{t}\left[\frac{\sigma^{2}}{2} \Delta v-(v \cdot \nabla) v-\nabla p+f(s, x)\right] d s+\int_{t_{k}}^{t} \sum_{r=1}^{q} \gamma_{r}(s, x) d w_{r}(s) .
\end{aligned}
$$

Then it is not difficult to obtain the estimate (3.8) using (2.17) and the assumptions on $f$ and $\gamma_{r}$.

$$
\begin{aligned}
& \text { From (3.9) and (3.2), we have } \\
& ((v-\hat{v}) \cdot \nabla) \tilde{\omega} \\
= & \left(\int_{t_{k}}^{t}\left[\frac{\sigma^{2}}{2} \Delta v-(v \cdot \nabla) v-\nabla p+f(s, x)\right] d s . \nabla\right) \tilde{\omega}(t, x)+\left(\int_{t_{k}}^{t} \sum_{r=1}^{q} \gamma_{r}(s, x) d w_{r}(s) . \nabla\right) \\
& \times\left\{\tilde{\omega}\left(t_{k}, x\right)+\int_{t_{k}}^{t}\left[\frac{\sigma^{2}}{2} \Delta \tilde{\omega}-(\hat{v} \cdot \nabla) \tilde{\omega}+g(s, x)\right] d s+\int_{t_{k}}^{t} \sum_{r=1}^{q} \mu_{r}(s, x) d w_{r}(s)\right\},
\end{aligned}
$$

from which it is not difficult to see that the inequality (3.7) holds. Lemma 3.1 is proved.

Now we proceed to proving estimates for the one-step error of $\tilde{\omega}(t, x)$.

Theorem 3.1. Let Assumption 2.1 hold with $m=2$. The one-step error of $\tilde{\omega}(t, x), t_{k} \leq$ $t \leq t_{k+1}$, which solves (3.2)-(3.3), has the following bounds for $t_{k} \leq t \leq t_{k+1}$ and sufficiently small $h>0$ :

$$
\begin{aligned}
& \left\|E\left[\delta_{\omega}(t, \cdot) \mid \mathcal{F}_{t_{k}}\right]\right\| \leq C\left(t_{k}, \omega\right) h^{2} \\
& \left(E\left\|\delta_{\omega}(t, \cdot)\right\|^{2}\right)^{\frac{1}{2}} \leq K h^{\frac{3}{2}}
\end{aligned}
$$


where $C\left(t_{k}, \omega\right)>0$ is an $\mathcal{F}_{t_{k}}$-measurable random variable with moments of a sufficiently high order bounded by a constant independent of $h$ and $K>0$ is a constant independent of $h$.

Proof. Taking scalar product of (3.4) and $\delta_{\omega}(t, x)$, using integration by parts and the property $\operatorname{div} v(t, x)=0$, we get

$$
\begin{aligned}
\frac{1}{2} d\left\|\delta_{\omega}(t, \cdot)\right\|^{2}= & \frac{\sigma^{2}}{2}\left(\Delta \delta_{\omega}(t, \cdot), \delta_{\omega}(t, \cdot)\right) d t-\left((v(t, \cdot) \cdot \nabla) \delta_{\omega}(t, \cdot), \delta_{\omega}(t, \cdot)\right) d t \\
& -\left(((v(t, \cdot)-\hat{v}(\cdot)) \cdot \nabla) \tilde{\omega}(t, \cdot), \delta_{\omega}(t, \cdot)\right) d t \\
= & -\frac{\sigma^{2}}{2}\left\|\nabla \delta_{\omega}(t, \cdot)\right\|^{2} d t-\left(((v(t, \cdot)-\hat{v}(\cdot)) \cdot \nabla) \tilde{\omega}(t, \cdot), \delta_{\omega}(t, \cdot)\right) d t
\end{aligned}
$$

Then

$$
\begin{aligned}
& \frac{1}{2} d E\left\|\delta_{\omega}(t, \cdot)\right\|^{2} \\
= & -\frac{\sigma^{2}}{2} E\left\|\nabla \delta_{\omega}(t, \cdot)\right\|^{2} d t-E\left(((v(t, \cdot)-\hat{v}(\cdot)) \cdot \nabla) \tilde{\omega}(t, \cdot), \delta_{\omega}(t, \cdot)\right) d t .
\end{aligned}
$$

For the last term in (3.13), we get

$$
\begin{aligned}
& \left|E\left(((v(t, \cdot)-\hat{v}(\cdot)) \cdot \nabla) \tilde{\omega}(t, \cdot), \delta_{\omega}(t, \cdot)\right)\right| \\
\leq & K E\|v(t, \cdot)-\hat{v}(\cdot)\| \cdot\|\tilde{\omega}(t, \cdot)\|_{3} \cdot\left\|\delta_{\omega}(t, \cdot)\right\| \\
\leq & K\left(E\|v(t, \cdot)-\hat{v}(\cdot)\|^{2} \cdot\|\tilde{\omega}(t, \cdot)\|_{3}^{2}\right)^{\frac{1}{2}}\left(E\left\|\delta_{\omega}(t, \cdot)\right\|^{2}\right)^{\frac{1}{2}} \\
\leq & K\left(E\|v(t, \cdot)-\hat{v}(\cdot)\|^{4}\right)^{\frac{1}{4}}\left(E\|\tilde{\omega}(t, \cdot)\|_{3}^{4}\right)^{\frac{1}{4}}\left(E\left\|\delta_{\omega}(t, \cdot)\right\|^{2}\right)^{\frac{1}{2}} \\
\leq & K h^{\frac{1}{2}}\left(E\left\|\delta_{\omega}(t, \cdot)\right\|^{2}\right)^{\frac{1}{2}}
\end{aligned}
$$

where for the first line we used the inequality (2.12) with $m_{1}=0, m_{2}=2, m_{3}=0$. We applied the Cauchy-Bunyakovski inequality twice to arrive at the penultimate line in the Eq. 3.14 and we used the error estimate (3.8) with $p=2$ and boundedness of the moment $E\|\tilde{\omega}(t, \cdot)\|_{3}^{4}$ (see (3.6)) to obtain the last line.

Thus

$$
\frac{1}{2} d E\left\|\delta_{\omega}(t, \cdot)\right\|^{2} \leq K h^{\frac{1}{2}}\left(E\left\|\delta_{\omega}(t, \cdot)\right\|^{2}\right)^{\frac{1}{2}} d t
$$

and since $\delta_{\omega}\left(t_{k}, x\right)=0$, we arrive at

$$
\int_{t_{k}}^{t} \frac{1}{2} \frac{d E\left\|\delta_{\omega}(s, \cdot)\right\|^{2}}{\left(E\left\|\delta_{\omega}(s, \cdot)\right\|^{2}\right)^{\frac{1}{2}}}=\left(E\left\|\delta_{\omega}(t, \cdot)\right\|^{2}\right)^{\frac{1}{2}} \leq K h^{\frac{3}{2}}
$$

confirming (3.11).

Now we are to prove (3.10). Using (3.4), we write the equation for $d E\left[\delta_{\omega}(t, x) \mid \mathcal{F}_{t_{k}}\right]$ and, after taking scalar product of the components of this equation and $E\left[\delta_{\omega}(t, x) \mid \mathcal{F}_{t_{k}}\right]$ and doing integration by parts, we arrive 


$$
\begin{aligned}
& \frac{1}{2} d\left\|E\left[\delta_{\omega}(t, \cdot) \mid \mathcal{F}_{t_{k}}\right]\right\|^{2} \\
= & -\frac{\sigma^{2}}{2}\left\|\nabla E\left[\delta_{\omega}(t, \cdot) \mid \mathcal{F}_{t_{k}}\right]\right\|^{2} d t-\left(E\left[(v(t, \cdot) . \nabla) \delta_{\omega}(t, \cdot) \mid \mathcal{F}_{t_{k}}\right], E\left[\delta_{\omega}(t, \cdot) \mid \mathcal{F}_{t_{k}}\right]\right) d t \\
& -\left(E\left[((v(t, \cdot)-\hat{v}(\cdot)) . \nabla) \tilde{\omega}(t, \cdot) \mid \mathcal{F}_{t_{k}}\right], E\left[\delta_{\omega}(t, \cdot) \mid \mathcal{F}_{t_{k}}\right]\right) d t
\end{aligned}
$$

By (3.7), we get for the third term in (3.15):

$$
\begin{aligned}
& \left|\left(E\left[((v(t, \cdot)-\hat{v}(\cdot)) . \nabla) \tilde{\omega}(t, \cdot) \mid \mathcal{F}_{t_{k}}\right], E\left[\delta_{\omega}(t, \cdot) \mid \mathcal{F}_{t_{k}}\right]\right)\right| \\
\leq & \left\|E\left[((v(t, \cdot)-\hat{v}(\cdot)) . \nabla) \tilde{\omega}(t, \cdot) \mid \mathcal{F}_{t_{k}}\right]\right\| \cdot\left\|E\left[\delta_{\omega}(t, \cdot) \mid \mathcal{F}_{t_{k}}\right]\right\| \\
\leq & C\left(t_{k}, \omega\right) h\left\|E\left[\delta_{\omega}(t, \cdot) \mid \mathcal{F}_{t_{k}}\right]\right\|,
\end{aligned}
$$

where $C\left(t_{k}, \omega\right)>0$ is an $\mathcal{F}_{t_{k}}$-measurable random variable which has moments up to a sufficiently high order and does not depend on $h$.

By simple manipulations and using (2.12), $m_{1}=2, m_{2}=0, m_{3}=0$ as well as (2.26), the Cauchy-Bunyakovski inequality, (2.23), a conditional version of (3.11), and (3.6), we obtain for the second term in (3.15):

$$
\begin{aligned}
& \left|\left(E\left[(v(t, \cdot) \cdot \nabla) \delta_{\omega}(t, \cdot) \mid \mathcal{F}_{t_{k}}\right], E\left[\delta_{\omega}(t, \cdot) \mid \mathcal{F}_{t_{k}}\right]\right)\right| \\
= & \left|E\left[\left((v(t, \cdot) \cdot \nabla) \delta_{\omega}(t, \cdot), E\left[\delta_{\omega}(t, \cdot) \mid \mathcal{F}_{t_{k}}\right]\right) \mid \mathcal{F}_{t_{k}}\right]\right| \\
\leq & E\left[\left|\left((v(t, \cdot) \cdot \nabla) \delta_{\omega}(t, \cdot), E\left[\delta_{\omega}(t, \cdot) \mid \mathcal{F}_{t_{k}}\right]\right)\right| \mid \mathcal{F}_{t_{k}}\right] \\
= & E\left[\left|\left((v(t, \cdot) \cdot \nabla) E\left[\delta_{\omega}(t, \cdot) \mid \mathcal{F}_{t_{k}}\right], \delta_{\omega}(t, \cdot)\right)\right| \mid \mathcal{F}_{t_{k}}\right] \\
\leq & K E\left[\|v(t, \cdot)\|_{2} \cdot\left\|E\left[\delta_{\omega}(t, \cdot) \mid \mathcal{F}_{t_{k}}\right]\right\|_{1} \cdot\left\|\delta_{\omega}(t, \cdot)\right\| \mid \mathcal{F}_{t_{k}}\right] \\
= & K\left\|E\left[\delta_{\omega}(t, \cdot) \mid \mathcal{F}_{t_{k}}\right]\right\|_{1} E\left[\|v(t, \cdot)\|_{2} \cdot\left\|\delta_{\omega}(t, \cdot)\right\| \mid \mathcal{F}_{t_{k}}\right] \\
\leq & K\left\|\nabla E\left[\delta_{\omega}(t, \cdot) \mid \mathcal{F}_{t_{k}}\right]\right\| E\left[\|\omega(t, \cdot)\|_{1} \cdot\left\|\delta_{\omega}(t, \cdot)\right\| \mid \mathcal{F}_{t_{k}}\right] \\
\leq & K\left\|\nabla E\left[\delta_{\omega}(t, \cdot) \mid \mathcal{F}_{t_{k}}\right]\right\|\left(E\left[\|\omega(t, \cdot)\|_{1}^{2} \mid \mathcal{F}_{t_{k}}\right]\right)^{\frac{1}{2}}\left(E\left[\left\|\delta_{\omega}(t, \cdot)\right\|^{2} \mid \mathcal{F}_{t_{k}}\right]\right)^{\frac{1}{2}} \\
\leq & C\left(t_{k}, \omega\right) h^{\frac{3}{2}}\left\|\nabla E\left[\delta_{\omega}(t, \cdot) \mid \mathcal{F}_{t_{k}}\right]\right\|,
\end{aligned}
$$

where $C\left(t_{k}, \omega\right)>0$ is an $\mathcal{F}_{t_{k}}$-measurable random variable which has moments up to a sufficiently high order and does not depend on $h$.

Combining (3.15)-(3.17), we arrive at

$$
\begin{aligned}
& \frac{1}{2} d\left\|E\left[\delta_{\omega}(t, \cdot) \mid \mathcal{F}_{t_{k}}\right]\right\|^{2} \\
\leq & -\frac{\sigma^{2}}{2}\left\|\nabla E\left[\delta_{\omega}(t, \cdot) \mid \mathcal{F}_{t_{k}}\right]\right\|^{2} d t+C\left(t_{k}, \omega\right) h^{\frac{3}{2}}\left\|\nabla E\left[\delta_{\omega}(t, \cdot) \mid \mathcal{F}_{t_{k}}\right]\right\| d t \\
& +C\left(t_{k}, \omega\right) h\left\|E\left[\delta_{\omega}(t, \cdot) \mid \mathcal{F}_{t_{k}}\right]\right\| d t \\
= & -\frac{1}{2}\left(\sigma\left\|\nabla E\left[\delta_{\omega}(t, \cdot) \mid \mathcal{F}_{t_{k}}\right]\right\|-\frac{1}{\sigma} C\left(t_{k}, \omega\right) h^{\frac{3}{2}}\right)^{2} d t \\
& +\frac{1}{2 \sigma^{2}} C^{2}\left(t_{k}, \omega\right) h^{3} d t+C\left(t_{k}, \omega\right) h\left\|E\left[\delta_{\omega}(t, \cdot) \mid \mathcal{F}_{t_{k}}\right]\right\| d t
\end{aligned}
$$




$$
\leq \frac{1}{2 \sigma^{2}} C^{2}\left(t_{k}, \omega\right) h^{3} d t+C\left(t_{k}, \omega\right) h\left\|E\left[\delta_{\omega}(t, \cdot) \mid \mathcal{F}_{t_{k}}\right]\right\| d t .
$$

Then, for some $\mathcal{F}_{t_{k}}$-measurable independent of $h$ random variable $C\left(t_{k}, \omega\right)>0$, we have

$$
d\left\|E\left[\delta_{\omega}(t, \cdot) \mid \mathcal{F}_{t_{k}}\right]\right\|^{2} \leq C\left(t_{k}, \omega\right) h^{3} d t+\frac{1}{h}\left\|E\left[\delta_{\omega}(t, \cdot) \mid \mathcal{F}_{t_{k}}\right]\right\|^{2} d t
$$

from which (3.10) follows taking into account that $\left\|E\left[\delta_{\omega}\left(t_{k}, \cdot\right) \mid \mathcal{F}_{t_{k}}\right]\right\|=0$. Theorem 3.1 is proved.

We define

$$
\tilde{v}(t, x):=U \tilde{\omega}(t, x), \quad t_{k} \leq t \leq t_{k+1}
$$

where the operator $U$ is from (2.18).

We prove the following corollary to Theorem 3.1.

Corollary 3.1. The one-step error $\delta_{v}(t, x):=v(t, x)-\tilde{v}(t, x)=U \delta_{\omega}(t, x)$ of $v(t, x), t_{k} \leq$ $t \leq t_{k+1}$ has the following bounds for $t_{k} \leq t \leq t_{k+1}$ :

$$
\begin{aligned}
& \left\|E \delta_{v}(t, \cdot)\right\| \leq K h^{2}, \\
& \left(E\left\|\delta_{v}(t, \cdot)\right\|^{2}\right)^{\frac{1}{2}} \leq K h^{\frac{3}{2}},
\end{aligned}
$$

where $K>0$ is independent of $h$.

Proof. Let the Fourier coefficients for $\delta_{\omega}(t, x)$ be $\left(\delta_{\omega}(t, \cdot)\right)_{\mathbf{n}}$, i.e.

$$
\delta_{\omega}(t, x)=\sum\left(\delta_{\omega}(t, \cdot)\right)_{\mathbf{n}} e^{i \frac{2 \pi}{L}(\mathbf{n}, x)}
$$

Hence by (2.18) we get

$$
\delta_{v}(t, x)=\frac{i L}{2 \pi} \sum \frac{1}{|\mathbf{n}|^{2}} e^{i \frac{2 \pi}{L}(\mathbf{n}, x)}\left(\delta_{\omega}(t, \cdot)\right)_{\mathbf{n}}\left[\begin{array}{r}
n^{2} \\
-n^{1}
\end{array}\right]
$$

i.e., the Fourier coefficients for $\delta_{v}(t, x)$ are

$$
\left(\delta_{v}(t, \cdot)\right)_{\mathbf{n}}=\frac{i L}{2 \pi} \frac{1}{|\mathbf{n}|^{2}}\left(\delta_{\omega}(t, \cdot)\right)_{\mathbf{n}}\left[\begin{array}{r}
n^{2} \\
-n^{1}
\end{array}\right] .
$$

Then, by Parseval's identity (2.11), we have

$$
\begin{aligned}
\left\|\delta_{v}(t, \cdot)\right\|_{L^{2}} & =\int_{Q}\left|\delta_{v}(t, x)\right|^{2} d x=L^{2} \sum\left|\left(\delta_{v}(t, \cdot)\right)_{\mathbf{n}}\right|^{2} \\
& =\frac{L^{4}}{4 \pi^{2}} \sum\left|\left(\delta_{\omega}(t, \cdot)\right)_{\mathbf{n}}\right|^{2} \frac{\left(n^{1}\right)^{2}+\left(n^{2}\right)^{2}}{|\mathbf{n}|^{4}} \\
& =\frac{L^{4}}{4 \pi^{2}} \sum \frac{\left|\left(\delta_{\omega}(t, \cdot)\right)_{\mathbf{n}}\right|^{2}}{|\mathbf{n}|^{2}} \leq \frac{L^{4}}{4 \pi^{2}} \sum\left|\left(\delta_{\omega}(t, \cdot)\right)_{\mathbf{n}}\right|^{2}
\end{aligned}
$$




$$
=\frac{L^{2}}{4 \pi^{2}} \int_{Q}\left|\delta_{\omega}(t, x)\right|^{2} d x=\frac{L^{2}}{4 \pi^{2}}\left\|\delta_{\omega}(t, \cdot)\right\|_{L^{2}},
$$

which together with (3.11) implies (3.20).

Let us now prove (3.19). We have

$$
E\left(\delta_{v}(t, \cdot)\right)_{\mathbf{n}}=\frac{i L}{2 \pi} \frac{1}{|\mathbf{n}|^{2}} E\left(\delta_{\omega}(t, \cdot)\right)_{\mathbf{n}}\left[\begin{array}{r}
n^{2} \\
-n^{1}
\end{array}\right]
$$

and hence

$$
\begin{aligned}
\left\|E \delta_{v}(t, \cdot)\right\|_{L^{2}} & =\int_{Q}\left|E \delta_{v}(t, x)\right|^{2} d x=L^{2} \sum\left|E\left(\delta_{v}(t, \cdot)\right)_{\mathbf{n}}\right|^{2} \\
& =\frac{L^{4}}{4 \pi^{2}} \sum\left|E\left(\delta_{\omega}(t, \cdot)\right)_{\mathbf{n}}\right|^{2} \frac{\left(n^{1}\right)^{2}+\left(n^{2}\right)^{2}}{|\mathbf{n}|^{4}} \\
& \leq \frac{L^{2}}{4 \pi^{2}}\left\|E \delta_{\omega}(t, \cdot)\right\|_{L^{2}},
\end{aligned}
$$

which together with (3.10) implies (3.19). Corollary 3.1 is proved.

\section{The method}

Based on the one-step approximation (3.1)-(3.3), we construct the global approximation $\tilde{\omega}(t, x)$ for SNSE (2.19)-(2.21) as follows.

On the first step of the method we set

$$
\begin{aligned}
& \tilde{\omega}\left(t_{0}, x\right)=\operatorname{curl} v\left(t_{0}, x\right)=\phi(x)=\operatorname{curl} \varphi(x), \\
& \hat{v}_{0}(x):=v\left(t_{0}, x\right)=\varphi(x) .
\end{aligned}
$$

Then we solve the linear SPDE (3.2)-(3.3) with $\hat{v}(x)=\hat{v}_{0}(x)$ on $\left[t_{0}, t_{1}\right]$ to obtain $\tilde{\omega}(t, x)$ and to construct

$$
\hat{v}_{1}(x)=U \tilde{\omega}\left(t_{1}, x\right) .
$$

On the second step we solve (3.2)-(3.3) on $\left[t_{1}, t_{2}\right]$ having $\tilde{\omega}\left(t_{1}, x\right)$ and setting $\hat{v}(x)=$ $\hat{v}_{1}(x)$. As a result, we obtain $\tilde{\omega}(t, x)$ on $\left[t_{1}, t_{2}\right]$ and $\hat{v}_{2}(x)=U \tilde{\omega}\left(t_{2}, x\right)$, and so on. Proceeding in this way, we obtain on the $N$-th step the approximation $\tilde{\omega}(t, x)$ on $\left[t_{N-1}, t_{N}\right]$ for $\omega(t, x)$ having $\tilde{\omega}\left(t_{N-1}, x\right)$ and $\hat{v}(x)=\hat{v}_{N-1}(x)=U \tilde{\omega}\left(t_{N-1}, x\right)$. Finally, $\hat{v}_{N}(x)=U \tilde{\omega}(T, x)$ which approximates the velocity $v(T, x)$.

In order to realise the approximation process described above, it is sufficient that on every time interval $\left[t_{k}, t_{k+1}\right], k=0, \ldots, N-1$, there exists a solution of the linear SPDE (3.2)-(3.3). We denote this solution as $\tilde{\omega}_{k}(t, x)$. It satisfies the condition

$$
\tilde{\omega}_{k}\left(t_{k}, x\right)= \begin{cases}\operatorname{curl} \varphi(x), & k=0, \\ \tilde{\omega}_{k-1}\left(t_{k}, x\right), & k=1, \ldots, N,\end{cases}
$$


and the time-independent coefficient $\hat{v}(x)$ in (3.2)-(3.3) is defined within each interval $\left(t_{k}, t_{k+1}\right]$ as

$$
\hat{v}(x):=\hat{v}_{k}(x):=U \tilde{\omega}_{k}\left(t_{k}, x\right), \quad t_{k}<t \leq t_{k+1} .
$$

Clearly, $\hat{v}(x)$ used in (3.2) are different on the time intervals $\left(t_{k}, t_{k+1}\right]$.

Before considering the global error of the approximation in Section 5, we now prove boundedness of the approximation's moments.

Theorem 4.1. Let Assumption 2.1 hold with $m=0$. The moments of the global approximation $\tilde{\omega}_{k}\left(t_{k}, x\right)$ and $\hat{v}_{k}(x)$ are uniformly bounded in $h$ and $k$ :

$$
\begin{aligned}
& E\left\|\tilde{\omega}_{k}\left(t_{k+1}, \cdot\right)\right\|^{2 p} \leq\|\phi(\cdot)\|^{2 p}+K \int_{0}^{t_{k+1}}\left(\|g(s, \cdot)\|^{2 p}+\sum_{r=1}^{q}\left\|\mu_{r}(s, \cdot)\right\|^{2 p}\right) d s, \\
& E\left\|\hat{v}_{k}(\cdot)\right\|^{2 p} \leq K E\left\|\tilde{\omega}_{k}\left(t_{k}, \cdot\right)\right\|^{2 p}
\end{aligned}
$$

where $K>0$ is independent of $h$ and $t_{k}$ but depends on $p \geq 1$.

Proof. For every sufficiently large integer $n$, define the stopping time

$$
\tau_{n}=\inf \left\{0<t \leq T:\|\tilde{\omega}(t, \cdot)\|^{2} \geq n\right\} .
$$

Using the Ito formula, doing integration by parts and taking into account that $\hat{v}_{k}(x)$ is divergence free, we obtain

$$
\begin{aligned}
& d\left\|\tilde{\omega}_{k}(t, \cdot)\right\|^{2 p} \\
= & 2 p\left\|\tilde{\omega}_{k}(t, \cdot)\right\|^{2(p-1)}\left[-\frac{\sigma^{2}}{2}\left\|\nabla \tilde{\omega}_{k}(t, \cdot)\right\|^{2}+\left(g(t, \cdot), \tilde{\omega}_{k}(t, \cdot)\right)+\frac{2 p-1}{2} \sum_{r=1}^{q}\left\|\mu_{r}(t, \cdot)\right\|^{2}\right] d t \\
& +2 p\left\|\tilde{\omega}_{k}(t, \cdot)\right\|^{2(p-1)} \sum_{r=1}^{q}\left(\mu_{r}(t, \cdot), \tilde{\omega}_{k}(t, \cdot)\right) d w_{r}(t), \quad t_{k} \wedge \tau_{n} \leq t \leq t_{k+1} \wedge \tau_{n}, \\
& \left\|\tilde{\omega}_{k}\left(t_{k}, \cdot\right)\right\|^{2 p}=\left\|\tilde{\omega}_{k-1}\left(t_{k}, \cdot\right)\right\|^{2 p} .
\end{aligned}
$$

We have

$$
\begin{aligned}
& d E\left\|\tilde{\omega}_{k}\left(t \wedge \tau_{n}, \cdot\right)\right\|^{2 p} \\
& =2 p\left[-\frac{\sigma^{2}}{2} E\left(\left\|\tilde{\omega}_{k}\left(t \wedge \tau_{n}, \cdot\right)\right\|^{2(p-1)}\left\|\nabla \tilde{\omega}_{k}\left(t \wedge \tau_{n}, \cdot\right)\right\|^{2}\right)\right. \\
& \quad+E\left(\left\|\tilde{\omega}_{k}\left(t \wedge \tau_{n}, \cdot\right)\right\|^{2(p-1)}\left(g\left(t \wedge \tau_{n}, \cdot\right), \tilde{\omega}_{k}\left(t \wedge \tau_{n}, \cdot\right)\right)\right) \\
& \left.\quad+\frac{2 p-1}{2} E\left\|\tilde{\omega}_{k}\left(t \wedge \tau_{n}, \cdot\right)\right\|^{2(p-1)} \sum_{r=1}^{q}\left\|\mu_{r}\left(t \wedge \tau_{n}, \cdot\right)\right\|^{2}\right] d t, \quad t_{k} \leq t \leq t_{k+1}, \\
& E\left\|\tilde{\omega}_{k}\left(t_{k} \wedge \tau_{n}, \cdot\right)\right\|^{2}=E\left\|\tilde{\omega}_{k-1}\left(t_{k} \wedge \tau_{n}, \cdot\right)\right\|^{2} .
\end{aligned}
$$


By Poincare's inequality (2.16) and doing simple re-arrangements, we arrive at

$$
\begin{aligned}
& d E\left\|\tilde{\omega}_{k}\left(t \wedge \tau_{n}, \cdot\right)\right\|^{2 p} \\
& \leq 2 p\left[-\alpha \frac{\sigma^{2}}{2} E\left\|\tilde{\omega}_{k}\left(t \wedge \tau_{n}, \cdot\right)\right\|^{2 p}+\alpha \frac{\sigma^{2}}{4} E\left\|\tilde{\omega}_{k}\left(t \wedge \tau_{n}, \cdot\right)\right\|^{2 p}+\frac{1}{\alpha \sigma^{2}}\left\|g\left(t \wedge \tau_{n}, \cdot\right)\right\|^{2}\right. \\
& \left.\quad+\alpha \frac{\sigma^{2}}{4} E\left\|\tilde{\omega}_{k}\left(t \wedge \tau_{n}, \cdot\right)\right\|^{2 p}+\frac{2[(2 p-1)(p-1)]^{p}}{\left(\alpha \sigma^{2}\right)^{p-1}(p-1)}\left[\sum_{r=1}^{q}\left\|\mu_{r}\left(t \wedge \tau_{n}, \cdot\right)\right\|^{2}\right]^{p}\right] d t .
\end{aligned}
$$

We note that the constant $\alpha>0$ in the above expression is due to Poincare's inequality (2.16) and it is, of course, independent of $h$ and $k$. Hence

$$
d E\left\|\tilde{\omega}_{k}\left(t \wedge \tau_{n}, \cdot\right)\right\|^{2 p} \leq K\left[\left\|g\left(t \wedge \tau_{n}, \cdot\right)\right\|^{2}+\left[\sum_{r=1}^{q}\left\|\mu_{r}\left(t \wedge \tau_{n}, \cdot\right)\right\|^{2}\right]^{p}\right] d t,
$$

where the constant $K>0$ depends on $p$ but independent of $h$ and $k$. The previous inequality implies

$$
\begin{aligned}
& E\left\|\tilde{\omega}_{k}\left(t_{k+1} \wedge \tau_{n}, \cdot\right)\right\|^{2 p} \\
\leq & E\left\|\tilde{\omega}_{k-1}\left(t_{k} \wedge \tau_{n}, \cdot\right)\right\|^{2 p}+K E \int_{t_{k} \wedge \tau_{n}}^{t_{k+1} \wedge \tau_{n}}\left(\|g(s, \cdot)\|^{2}+\left[\sum_{r=1}^{q}\left\|\mu_{r}(s, \cdot)\right\|^{2}\right]^{p}\right) d s \\
\leq & E\|\phi(\cdot)\|^{2 p}+E \int_{0}^{t_{k+1} \wedge \tau_{n}}\left(K\|g(s, \cdot)\|^{2}+\sum_{r=1}^{q}\left\|\mu_{r}(s, \cdot)\right\|^{2}\right) d s,
\end{aligned}
$$

and letting $n \rightarrow \infty$ we arrive at (4.3). The estimate (4.4) is an evident consequence of (4.3) (cf. e.g. (2.18)). Theorem 4.1 is proved.

Remark 4.1. It is not difficult to see that repeating the proof of Lemma 2.1 word by word, we can get that the exponential moment for $\left\|\tilde{\omega}_{k}\left(t_{k+1}, \cdot\right)\right\|^{2}$ is bounded, more precisely, the estimate of the form (2.28) holds for $\left\|\tilde{\omega}_{k}\left(t_{k+1}, \cdot\right)\right\|^{2}$ under Assumption 2.1 with $m=0$.

Now we consider uniform bounds for moments of higher Sobolev norms of $\tilde{\omega}_{k}$.

Theorem 4.2. Let Assumption 2.1 hold with $m>0$. Then for $p \geq 1$

$$
E\left\|\tilde{\omega}_{k}\left(t_{k+1}, \cdot\right)\right\|_{m}^{2 p} \leq\|\phi(\cdot)\|_{m}^{2 p}+K t_{k+1}
$$

where $K>0$ is independent of $h$ and $t_{k}$.

Proof. The proof is by induction. To this end, we assume that for all $l \leq m-1$ the moments $E\left\|\tilde{\omega}_{k}(t, \cdot)\right\|_{l}^{2 p}$ are bounded (uniformly in $k$ and $h$ ) and for sufficiently large $p \geq 1$ (note that Theorem 4.1 guarantees their boundedness for $m=0$ ). 
We will be adapting recipes from [28, Section 4.1] and [16, Section 3.4]. Let the operator $\Lambda$ be such that $\Lambda^{2}=-\Delta$. We can obtain for an integer $m \geq 1$ :

$$
\begin{aligned}
d\left\|\tilde{\omega}_{k}(t, \cdot)\right\|_{m}^{2}= & {\left[-\frac{\sigma^{2}}{2}\left\|\tilde{\omega}_{k}(t, \cdot)\right\|_{m+1}^{2}+\frac{\sigma^{2}}{2}\left\|\tilde{\omega}_{k}(t, \cdot)\right\|^{2}+\left(\tilde{\omega}_{k}(t, \cdot), g(t, \cdot)\right)_{m}\right.} \\
& \left.-\sum_{j=1}^{m}\left(\left(\hat{v}_{k}(\cdot) \cdot \nabla\right) \tilde{\omega}_{k}(t, \cdot), \Lambda^{2 j} \tilde{\omega}_{k}(t, \cdot)\right)\right] d t \\
& +\sum_{r=1}^{q}\left(\mu_{r}(t, \cdot), \tilde{\omega}_{k}(t, \cdot)\right)_{m} d w_{r}(t), \quad t_{k} \wedge \tau_{n} \leq t \leq t_{k+1} \wedge \tau_{n}, \\
\left\|\tilde{\omega}_{k}\left(t_{k}, \cdot\right)\right\|_{m}^{2}= & \left\|\tilde{\omega}_{k-1}\left(t_{k}, \cdot\right)\right\|_{m}^{2} .
\end{aligned}
$$

Here $\tau_{n}$ is

$$
\tau_{n}=\inf \left\{0<t \leq T:\|\tilde{\omega}(t, \cdot)\|_{m}^{2} \geq n\right\} .
$$

Then for $m \geq 1$ and $p \geq 1$, we have

$$
\begin{aligned}
& d\left\|\tilde{\omega}_{k}(t, \cdot)\right\|_{m}^{2 p} \\
& =2 p\left\|\tilde{\omega}_{k}(t, \cdot)\right\|_{m}^{2(p-1)}\left[-\frac{\sigma^{2}}{2}\left\|\tilde{\omega}_{k}(t, \cdot)\right\|_{m+1}^{2}+\frac{\sigma^{2}}{2}\left\|\tilde{\omega}_{k}(t, \cdot)\right\|^{2}+\left(\tilde{\omega}_{k}(t, \cdot), g(t, \cdot)\right)_{m}\right. \\
& \left.-\sum_{j=1}^{m}\left(\left(\hat{v}_{k}(\cdot) . \nabla\right) \tilde{\omega}_{k}(t, \cdot), \Lambda^{2 j} \tilde{\omega}_{k}(t, \cdot)\right)+\frac{2 p-1}{2} \sum_{r=1}^{q}\left\|\mu_{r}(t, \cdot)\right\|_{m}^{2}\right] d t \\
& +2 p\left\|\tilde{\omega}_{k}(t, \cdot)\right\|_{m}^{2(p-1)} \sum_{r=1}^{q}\left(\mu_{r}(t, \cdot), \tilde{\omega}_{k}(t, \cdot)\right)_{m} d w_{r}(t), \quad t_{k} \wedge \tau_{n} \leq t \leq t_{k+1} \wedge \tau_{n}, \\
& \left\|\tilde{\omega}_{k}\left(t_{k}, \cdot\right)\right\|_{m}^{2 p}=\left\|\tilde{\omega}_{k-1}\left(t_{k}, \cdot\right)\right\|_{m}^{2 p} .
\end{aligned}
$$

Let us analyze terms in the right-hand side of (4.7). We obtain for some $K>0$ dependent on $p$ :

$$
\frac{\sigma^{2}}{2}\left\|\tilde{\omega}_{k}(t, \cdot)\right\|_{m}^{2(p-1)}\left\|\tilde{\omega}_{k}(t, \cdot)\right\|^{2} \leq \frac{\sigma^{2}}{16}\left\|\tilde{\omega}_{k}(t, \cdot)\right\|_{m}^{2 p}+K\left\|\tilde{\omega}_{k}(t, \cdot)\right\|^{2 p} .
$$

We have (e.g. see [28, Eq. (4.4)]):

$$
\begin{aligned}
\left|\left(\tilde{\omega}_{k}(t, \cdot), g(t, \cdot)\right)_{m}\right| & \leq\|g(t, \cdot)\|_{m-1}\left\|\tilde{\omega}_{k}(t, \cdot)\right\|_{m+1} \\
& \leq \frac{8}{\sigma^{2}}\|g(t, \cdot)\|_{m-1}^{2}+\frac{\sigma^{2}}{8}\left\|\tilde{\omega}_{k}(t, \cdot)\right\|_{m+1}^{2}
\end{aligned}
$$

and

$$
K\left\|\tilde{\omega}_{k}(t, \cdot)\right\|_{m}^{2(p-1)}\|g(t, \cdot)\|_{m-1}^{2} \leq \frac{K^{p}}{p}\left(\frac{16 p}{\sigma^{2}(p-1)}\right)^{p-1}\|g(t, \cdot)\|_{m-1}^{2 p}+\frac{\sigma^{2}}{16}\left\|\tilde{\omega}_{k}(t, \cdot)\right\|_{m}^{2 p},
$$


hence

$$
\begin{aligned}
& \left|\left\|\tilde{\omega}_{k}(t, \cdot)\right\|_{m}^{2(p-1)}\left(\tilde{\omega}_{k}(t, \cdot), g(t, \cdot)\right)_{m}\right| \\
\leq & K\|g(t, \cdot)\|_{m-1}^{2 p}+\frac{\sigma^{2}}{16}\left\|\tilde{\omega}_{k}(t, \cdot)\right\|_{m}^{2 p}+\frac{\sigma^{2}}{8}\left\|\tilde{\omega}_{k}(t, \cdot)\right\|_{m}^{2(p-1)}\left\|\tilde{\omega}_{k}(t, \cdot)\right\|_{m+1}^{2} .
\end{aligned}
$$

Also, for some $K>0$ dependent on $p$ :

$$
\begin{aligned}
& \frac{2 p-1}{2}\left\|\tilde{\omega}_{k}(t, \cdot)\right\|_{m}^{2(p-1)} \sum_{r=1}^{q}\left\|\mu_{r}(t, \cdot)\right\|_{m}^{2} \\
\leq & K\left(\sum_{r=1}^{q}\left\|\mu_{r}(t, \cdot)\right\|_{m}^{2}\right)^{p}+\frac{\sigma^{2}}{16}\left\|\tilde{\omega}_{k}(t, \cdot)\right\|_{m}^{2 p} .
\end{aligned}
$$

Let us now estimate the trilinear-form:

$$
\begin{aligned}
& \sum_{j=1}^{m}\left|\left(\left(\hat{v}_{k}(\cdot) . \nabla\right) \tilde{\omega}_{k}(t, \cdot), \Lambda^{2 j} \tilde{\omega}_{k}(t, \cdot)\right)\right| \\
\leq & K \sum_{j=1}^{m} \sum_{\left[\alpha_{j}\right]=j} \sum_{l=1}^{j} \sum_{\substack{\left[\alpha_{l}\right]=l \\
\left[\alpha_{j-l}\right]=j-l}}\left|\left(\left(D^{\alpha_{l}} \hat{v}_{k}(\cdot) \cdot \nabla\right) D^{\alpha_{j-l}} \tilde{\omega}_{k}(t, \cdot), D^{\alpha_{j}} \tilde{\omega}_{k}(t, \cdot)\right)\right| \\
\leq & K \sum_{j=1}^{m} \sum_{\left[\alpha_{j}\right]=j} \sum_{l=1}^{j} \sum_{\substack{\left[\alpha_{l}\right]=l \\
\left[\alpha_{j-l}\right]=j-l}}\left\|D^{\alpha_{l}} \hat{v}_{k}(\cdot)\right\|\left\|D^{\alpha_{j-l}} \tilde{\omega}_{k}(t, \cdot)\right\|_{\frac{3}{2}}\left\|D^{\alpha_{j}} \tilde{\omega}_{k}(t, \cdot)\right\|_{1} \\
\leq & K \sum_{j=1}^{m} \sum_{l=1}^{j}\left\|\hat{v}_{k}(\cdot)\right\|\left\|_{l}\right\| \tilde{\omega}_{k}(t, \cdot)\left\|_{j-l+\frac{3}{2}}\right\| \tilde{\omega}_{k}(t, \cdot) \|_{j+1} \\
\leq & K\left\|\tilde{\omega}_{k}\left(t_{k}, \cdot\right)\right\|_{m-1}\left\|\tilde{\omega}_{k}(t, \cdot)\right\|_{m+\frac{1}{2}}\left\|\tilde{\omega}_{k}(t, \cdot)\right\|_{m+1} \\
\leq & K\left\|\tilde{\omega}_{k}(t, \cdot)\right\|_{m-1}^{\frac{5}{4}}\left\|\tilde{\omega}_{k}(t, \cdot)\right\|_{m+1}^{\frac{7}{4}} \\
\leq & \frac{K^{8}}{8}\left(\frac{56}{\sigma^{2}}\right)^{7}\left\|\tilde{\omega}_{k}\left(t_{k}, \cdot\right)\right\|_{m-1}^{10}+\frac{\sigma^{2}}{8}\left\|\tilde{\omega}_{k}(t, \cdot)\right\|_{m+1}^{2},
\end{aligned}
$$

where for the second line we used integration by parts (see this recipe in [28, pp. 2930]); for the third - the inequality (2.12) with $m_{1}=0, m_{1}=\frac{1}{2}, m_{3}=1$; for the fourth line - (2.6); for the fifth line - (2.24) and that $\|u(\cdot)\|_{m_{1}} \leq\|u(\cdot)\|_{m_{2}}$ for $m_{2} \geq m_{1}$; the sixth line is obtained using (2.13); and the final line follows from Young's inequality. Note that the constants $K>0$ are changing from line to line. Further,

$$
\begin{aligned}
& K\left\|\tilde{\omega}_{k}(t, \cdot)\right\|_{m-1}^{10}\left\|\tilde{\omega}_{k}(t, \cdot)\right\|_{m}^{2(p-1)} \\
\leq & \frac{K^{p}}{p}\left(\frac{8 p}{\alpha \sigma^{2}(p-1)}\right)^{p-1}\left\|\tilde{\omega}_{k}\left(t_{k}, \cdot\right)\right\|_{m-1}^{10 p}+\frac{\sigma^{2}}{16}\left\|\tilde{\omega}_{k}(t, \cdot)\right\|_{m}^{2 p} .
\end{aligned}
$$


Combining (4.7) with (4.8)-(4.12), we can write for some $K>0$ :

$$
\begin{aligned}
d\left\|\tilde{\omega}_{k}(t, \cdot)\right\|_{m}^{2 p} \leq 2 p[ & -\frac{\sigma^{2}}{4}\left\|\tilde{\omega}_{k}(t, \cdot)\right\|_{m}^{2(p-1)}\left\|\tilde{\omega}_{k}(t, \cdot)\right\|_{m+1}^{2}+\frac{\sigma^{2}}{4}\left\|\tilde{\omega}_{k}(t, \cdot)\right\|_{m}^{2 p} \\
& +K\left\|\tilde{\omega}_{k}(t, \cdot)\right\|^{2 p}+K\|g(t, \cdot)\|_{m-1}^{2 p} \\
& \left.+K\left(\sum_{r=1}^{q}\left\|\mu_{r}(t, \cdot)\right\|_{m}^{2}\right)^{p}+K E\left\|\tilde{\omega}_{k}\left(t_{k}, \cdot\right)\right\|_{m-1}^{10 p}\right] d t \\
& +2 p\left\|\tilde{\omega}_{k}(t, \cdot)\right\|_{m}^{2(p-1)} \sum_{r=1}^{q}\left(\mu_{r}(t, \cdot), \tilde{\omega}_{k}(t, \cdot)\right)_{m} d w_{r}(t) \quad t_{k} \wedge \tau_{n} \leq t \leq t_{k+1} \wedge \tau_{n}
\end{aligned}
$$

Thus (note that $-\left\|\tilde{\omega}_{k}(t, \cdot)\right\|_{m+1}^{2} \leq-\left\|\tilde{\omega}_{k}(t, \cdot)\right\|_{m}^{2}$ ), for some $K>0$

$$
\begin{aligned}
& \quad d E\left\|\tilde{\omega}_{k}\left(t \wedge \tau_{n}, \cdot\right)\right\|_{m}^{2 p} \\
& \leq K\left[E\left\|\tilde{\omega}_{k}\left(t \wedge \tau_{n}, \cdot\right)\right\|^{2 p}+\left\|g\left(t \wedge \tau_{n}, \cdot\right)\right\|_{m-1}^{2 p}+E\left\|\tilde{\omega}_{k}\left(t_{k} \wedge \tau_{n}, \cdot\right)\right\|_{m-1}^{10 p}\right. \\
& \left.\quad+\left(\sum_{r=1}^{q}\left\|\mu_{r}\left(t \wedge \tau_{n}, \cdot\right)\right\|_{m}^{2}\right)^{p}\right] d t, \quad t_{k} \leq t \leq t_{k+1}, \\
& E\left\|\tilde{\omega}_{k}\left(t_{k} \wedge \tau_{n}, \cdot\right)\right\|_{m}^{2 p}=E\left\|\tilde{\omega}_{k-1}\left(t_{k} \wedge \tau_{n}, \cdot\right)\right\|_{m}^{2 p} .
\end{aligned}
$$

By Assumption 2.1 and the induction assumption at the start of the proof, we get

$$
\begin{aligned}
& d E\left\|\tilde{\omega}_{k}\left(t \wedge \tau_{n}, \cdot\right)\right\|_{m}^{2 p} \leq K d t, \quad t_{k} \leq t \leq t_{k+1}, \\
& E\left\|\tilde{\omega}_{k}\left(t_{k} \wedge \tau_{n}, \cdot\right)\right\|_{m}^{2 p}=E\left\|\tilde{\omega}_{k-1}\left(t_{k} \wedge \tau_{n}, \cdot\right)\right\|_{m}^{2 p}, \\
& E\left\|\tilde{\omega}_{k}\left(t_{k+1} \wedge \tau_{n}, \cdot\right)\right\|_{m}^{2 p} \leq E\left\|\tilde{\omega}_{k-1}\left(t_{k} \wedge \tau_{n}, \cdot\right)\right\|_{m}^{2 p}+K h,
\end{aligned}
$$

from which (4.6) follows by the standard arguments. Theorem 4.2 is proved.

\section{Mean-square convergence theorem}

To prove the global convergence of $\tilde{\omega}_{k}\left(t_{k}, \cdot\right)$, we use the idea of the proof of the fundamental theorem of mean-square convergence for SDEs [21] (see also [23, Section 1.1]).

Theorem 5.1. Let Assumption 2.1 hold with $m=3$. The global approximation $\tilde{\omega}_{k}\left(t_{k+1}, x\right)$ for the problem (2.19)-(2.21) has the first mean-square order accuracy, i.e., for a sufficiently small $h>0$

$$
\left(E\left\|\omega\left(t_{k+1}, \cdot\right)-\tilde{\omega}_{k}\left(t_{k+1}, \cdot\right)\right\|^{2}\right)^{\frac{1}{2}} \leq K h, \quad k=0, \ldots, N-1,
$$

where $K>0$ is a constant independent of $k$ and $h$. 
Proof. We note that in the proof we shall again use letters $K$ and $C(\cdot, \omega)$ to denote various deterministic constants and random variables, respectively, which are independent of $h$ and $k$; their values may change from line to line.

We have

$$
\begin{aligned}
R\left(t_{k+1}, x\right):= & \omega\left(t_{k+1}, x\right)-\tilde{\omega}_{k}\left(t_{k+1}, x\right) \\
= & \omega\left(t_{k+1}, x ; 0, \phi\right)-\tilde{\omega}_{k}\left(t_{k+1}, x ; 0, \phi\right) \\
= & \omega\left(t_{k+1}, x ; t_{k}, \omega\left(t_{k}, \cdot\right)\right)-\tilde{\omega}_{k}\left(t_{k+1}, x ; t_{k}, \tilde{\omega}_{k}\left(t_{k}, \cdot\right)\right) \\
= & \left(\omega\left(t_{k+1}, x ; t_{k}, \omega\left(t_{k}, \cdot\right)\right)-\omega\left(t_{k+1}, x ; t_{k}, \tilde{\omega}_{k}\left(t_{k}, \cdot\right)\right)\right) \\
& +\left(\omega\left(t_{k+1}, x ; t_{k}, \tilde{\omega}_{k}\left(t_{k}, \cdot\right)\right)-\tilde{\omega}_{k}\left(t_{k+1}, x ; t_{k}, \tilde{\omega}_{k}\left(t_{k}, \cdot\right)\right)\right),
\end{aligned}
$$

where reflects function dependence of solutions on the initial conditions. The first difference in the right-hand side of (5.1) is the error of the solution arising due to the error in the initial data at time $t_{k}$, accumulated at the $k$-th step. The second difference is the one-step error at the $(k+1)$-step:

$$
\delta_{\omega}\left(t_{k+1}, x\right):=\omega\left(t_{k+1}, x ; t_{k}, \tilde{\omega}_{k}\left(t_{k}, \cdot\right)\right)-\tilde{\omega}_{k}\left(t_{k+1}, x ; t_{k}, \tilde{\omega}_{k}\left(t_{k}, \cdot\right)\right),
$$

for which estimates are given in Theorem 3.1 taking into account that Theorems 4.1 and 4.2 guarantees boundedness of moments of $\left\|\tilde{\omega}_{k}\left(t_{k}, \cdot\right)\right\|_{3}$ under the conditions of this theorem. Taking the $L^{2}$-norm of both sides of (5.1), we obtain

$$
\begin{aligned}
\left\|R\left(t_{k+1}, \cdot\right)\right\|^{2}= & \left\|\omega\left(t_{k+1}, \cdot ; t_{k}, \omega\left(t_{k}, \cdot\right)\right)-\omega\left(t_{k+1}, \cdot ; t_{k}, \tilde{\omega}_{k}\left(t_{k}, \cdot\right)\right)\right\|^{2}+\left\|\delta_{\omega}\left(t_{k+1}, \cdot\right)\right\|^{2} \\
& +2\left(\omega\left(t_{k+1}, \cdot ; t_{k}, \omega\left(t_{k}, \cdot\right)\right)-\omega\left(t_{k+1}, \cdot ; t_{k}, \tilde{\omega}_{k}\left(t_{k}, \cdot\right)\right), \delta_{\omega}\left(t_{k+1}, \cdot\right)\right),
\end{aligned}
$$

where the first - in each $\omega$ or $\tilde{\omega}_{k}$ reflects that we took $L^{2}$-norm.

Using (3.11) from Theorem 3.1 together with Theorems 4.1 and 4.2, we obtain for the second term in (5.3):

$$
\left\|\delta_{\omega}\left(t_{k+1}, \cdot\right)\right\|^{2} \leq C\left(t_{k+1}, \omega\right) h^{3}
$$

where $C\left(t_{k+1}, \omega\right)>0$ is an $\mathcal{F}_{t_{k+1}}$-measurable with bounded second moment.

By (2.38) from Lemma 2.2 together with Theorems 4.1 and 4.2, we get for the first term in (5.3):

$$
\begin{aligned}
& \left\|\omega\left(t_{k+1}, x ; t_{k}, \omega\left(t_{k}, \cdot\right)\right)-\omega\left(t_{k+1}, x ; t_{k}, \tilde{\omega}_{k}\left(t_{k}, \cdot\right)\right)\right\|^{2} \\
\leq & \left\|R\left(t_{k}, \cdot\right)\right\|^{2} \exp \left(K h+c \int_{t_{k}}^{t_{k+1}}\left\|\nabla \omega\left(s^{\prime}, \cdot ; t_{k}, \omega\left(t_{k}, \cdot\right)\right)\right\|^{2} d s^{\prime}\right) .
\end{aligned}
$$

The difference $\omega\left(t_{k+1}, x ; t_{k}, \omega\left(t_{k}, \cdot\right)\right)-\omega\left(t_{k+1}, x ; t_{k}, \tilde{\omega}_{k}\left(t_{k}, \cdot\right)\right)$ in the last summand in (5.3) can be treated using (2.37) from Lemma 2.2:

$$
\omega\left(t_{k+1}, x ; t_{k}, \omega\left(t_{k}, \cdot\right)\right)-\omega\left(t_{k+1}, x ; t_{k}, \tilde{\omega}_{k}\left(t_{k}, \cdot\right)\right)=R\left(t_{k}, x\right)+\eta\left(t_{k}, x\right) .
$$


Using a conditional version of (3.11) from Theorem 3.1 and (2.39) from Lemma 2.2 together with Theorems 4.1 and 4.2, we get

$$
\begin{aligned}
\left|\left(\eta\left(t_{k}, \cdot\right), \delta_{\omega}\left(t_{k+1}, \cdot\right)\right)\right| & \leq\left\|\eta\left(t_{k}, \cdot\right)\right\|\left\|\delta_{\omega}\left(t_{k+1}, \cdot\right)\right\| \\
& \leq\left\|\eta\left(t_{k}, \cdot\right)\right\|^{2}+\frac{1}{4}\left\|\delta_{\omega}\left(t_{k+1}, \cdot\right)\right\|^{2} \\
& \leq h\left\|R\left(t_{k}, \cdot\right)\right\|^{2}+C\left(t_{k+1}, \omega\right) h^{3}
\end{aligned}
$$

where $C\left(t_{k+1}, \omega\right)>0$ is an $\mathcal{F}_{t_{k+1}}$-measurable with bounded second moment.

Combining the above, we arrive at

$$
\begin{aligned}
\left\|R\left(t_{k+1}, \cdot\right)\right\|^{2} \leq & \left\|R\left(t_{k}, \cdot\right)\right\|^{2} \exp \left(K h+c \int_{t_{k}}^{t_{k+1}}\left\|\nabla \omega\left(s^{\prime}, \cdot ; t_{k}, \omega\left(t_{k}, \cdot\right)\right)\right\|^{2} d s^{\prime}\right) \\
& +h\left\|R\left(t_{k}, \cdot\right)\right\|^{2}+\left(R\left(t_{k}, \cdot\right), \delta_{\omega}\left(t_{k+1}, \cdot\right)\right)+C\left(t_{k+1}, \omega\right) h^{3} .
\end{aligned}
$$

Since $\|R(0, \cdot)\|=0$, summing (5.7) from $k=0$ to $n$, we get

$$
\begin{aligned}
& \left\|R\left(t_{n+1}, \cdot\right)\right\|^{2} \\
\leq & \sum_{k=1}^{n}\left\|R\left(t_{k}, \cdot\right)\right\|^{2}\left[\exp \left(K h+c \int_{t_{k}}^{t_{k+1}}\left\|\nabla \omega\left(s^{\prime}, \cdot ; t_{k}, \omega\left(t_{k}, \cdot\right)\right)\right\|^{2} d s^{\prime}\right)-1+h\right] \\
& +h^{3} \sum_{k=0}^{n} C\left(t_{k+1}, \omega\right)+\sum_{k=1}^{n}\left(R\left(t_{k}, \cdot\right), \delta_{\omega}\left(t_{k+1}, \cdot\right)\right) \\
\leq & \sum_{k=1}^{n}\left\|R\left(t_{k}, \cdot\right)\right\|^{2}\left[\exp \left(K h+c \int_{t_{k}}^{t_{k+1}}\left\|\nabla \omega\left(s^{\prime}, \cdot ; t_{k}, \omega\left(t_{k}, \cdot\right)\right)\right\|^{2} d s^{\prime}\right)-1\right] \\
& +h^{3} \sum_{k=0}^{n} C\left(t_{k+1}, \omega\right)+\sum_{k=1}^{n}\left(R\left(t_{k}, \cdot\right), \delta_{\omega}\left(t_{k+1}, \cdot\right)\right) .
\end{aligned}
$$

From which, by a version of Gronwall's lemma (see, e.g. [1,14]), we obtain

$$
\begin{aligned}
& \left\|R\left(t_{n+1}, \cdot\right)\right\|^{2} \\
\leq & F_{n}+\sum_{k=1}^{n} F_{k-1}\left[\exp \left(K h+c \int_{t_{k}}^{t_{k+1}}\left\|\nabla \omega\left(s^{\prime}, \cdot ; t_{k}, \omega\left(t_{k}, \cdot\right)\right)\right\|^{2} d s^{\prime}\right)-1\right] \\
& \times \prod_{j=k+1}^{n} \exp \left(K h+c \int_{t_{j}}^{t_{j+1}}\left\|\nabla \omega\left(s^{\prime}, \cdot ; t_{k}, \omega\left(t_{k}, \cdot\right)\right)\right\|^{2} d s^{\prime}\right),
\end{aligned}
$$

where

$$
F_{k}:=h^{3} \sum_{j=0}^{k} C\left(t_{j+1}, \omega\right)+\sum_{j=1}^{k}\left(R\left(t_{j}, \cdot\right), \delta_{\omega}\left(t_{j+1}, \cdot\right)\right)
$$

We have

$$
\left\|R\left(t_{n+1}, \cdot\right)\right\|^{2} \leq F_{n}+\sum_{k=1}^{n} F_{k-1}\left[\exp \left(K h+c \int_{t_{k}}^{t_{k+1}}\left\|\nabla \omega\left(s^{\prime}, \cdot ; t_{k}, \omega\left(t_{k}, \cdot\right)\right)\right\|^{2} d s^{\prime}\right)-1\right]
$$




$$
\begin{aligned}
& \times \exp \left(K\left(t_{n+1}-t_{k+1}\right)+c \int_{t_{k+1}}^{t_{n+1}}\left\|\nabla \omega\left(s^{\prime}, \cdot ; t_{k+1}, \omega\left(t_{k+1}, \cdot\right)\right)\right\|^{2} d s^{\prime}\right) \\
&=F_{n}+\sum_{k=1}^{n} F_{k-1}\left[\exp \left(K\left(t_{n+1}-t_{k}\right)+c \int_{t_{k}}^{t_{n+1}}\left\|\nabla \omega\left(s^{\prime}, \cdot ; t_{k}, \omega\left(t_{k}, \cdot\right)\right)\right\|^{2} d s^{\prime}\right)\right. \\
&\left.\quad-\exp \left(K\left(t_{n+1}-t_{k+1}\right)+c \int_{t_{k+1}}^{t_{n+1}}\left\|\nabla \omega\left(s^{\prime}, \cdot ; t_{k+1}, \omega\left(t_{k+1}, \cdot\right)\right)\right\|^{2} d s^{\prime}\right)\right] \\
&=\sum_{k=1}^{n}\left(F_{k}-F_{k-1}\right) \exp \left(K\left(t_{n+1}-t_{k+1}\right)+c \int_{t_{k+1}}^{t_{n+1}}\left\|\nabla \omega\left(s^{\prime}, \cdot ; t_{k+1}, \omega\left(t_{k+1}, \cdot\right)\right)\right\|^{2} d s^{\prime}\right) \\
&+h^{3} C\left(t_{1}, \omega\right) \exp \left(K\left(t_{n+1}-t_{1}\right)+c \int_{t_{1}}^{t_{n+1}}\left\|\nabla \omega\left(s^{\prime}, \cdot ; t_{1}, \omega\left(t_{1}, \cdot\right)\right)\right\|^{2} d s^{\prime}\right) .
\end{aligned}
$$

For the last term in the right-hand side of (5.9), we obtain using the CauchyBunyakovski inequality and Lemma 2.1:

$$
\begin{aligned}
& E\left\{h^{3} C\left(t_{1}, \omega\right) \exp \left(K\left(t_{n+1}-t_{1}\right)+c \int_{t_{k+1}}^{t_{n+1}}\left\|\nabla \omega\left(s^{\prime}, \cdot ; 0, \phi(\cdot)\right)\right\|^{2} d s^{\prime}\right)\right\} \\
\leq & K h^{3} .
\end{aligned}
$$

Consider now the first term in the right-hand side of (5.9). We have

$$
\begin{aligned}
& \left(F_{k}-F_{k-1}\right) \exp \left(K\left(t_{n+1}-t_{k+1}\right)+c \int_{t_{k+1}}^{t_{n+1}}\left\|\nabla \omega\left(s^{\prime}, \cdot ; t_{k+1}, \omega\left(t_{k+1}, \cdot\right)\right)\right\|^{2} d s^{\prime}\right) \\
= & \exp \left(K\left(t_{n+1}-t_{k+1}\right)+c \int_{t_{k+1}}^{t_{n+1}}\left\|\nabla \omega\left(s^{\prime}, \cdot ; t_{k+1}, \omega\left(t_{k+1}, \cdot\right)\right)\right\|^{2} d s^{\prime}\right) \\
& \times\left[h^{3} C\left(t_{k+1}, \omega\right)+\left(R\left(t_{k}, \cdot\right), \delta_{\omega}\left(t_{k+1}, \cdot\right)\right)\right] .
\end{aligned}
$$

Expectation of the first term from the right-hand side of (5.11) is estimated by $K h^{3}$ as in (5.10).

Let us now consider the second term in (5.11). By the martingale representation theorem and Lemma 2.1, we can obtain

$$
\begin{aligned}
& E\left[\exp \left(K\left(t_{n+1}-t_{k+1}\right)+c \int_{t_{k+1}}^{t_{n+1}}\left\|\nabla \omega\left(s^{\prime}, \cdot ; t_{k+1}, \omega\left(t_{k+1}, \cdot\right)\right)\right\|^{2} d s^{\prime}\right) \mid \mathcal{F}_{t_{k+1}}\right] \\
= & E\left[\exp \left(K\left(t_{n+1}-t_{k+1}\right)+c \int_{t_{k+1}}^{t_{n+1}}\left\|\nabla \omega\left(s^{\prime}, \cdot ; t_{k+1}, \omega\left(t_{k+1}, \cdot\right)\right)\right\|^{2} d s^{\prime}\right)\right] \\
& +\sum_{r=1}^{q} \int_{0}^{t_{k+1}} \lambda_{r}(s) d w_{r}(s)
\end{aligned}
$$

where $\lambda_{r}(s)$ are $\mathcal{F}_{s}$-adapted square-integrable stochastic processes. 
Using (3.10) and a conditional version of (3.11) from Theorem 3.1 together with Theorems 4.1 and 4.2 and also using Lemma 2.1 and (5.12), we arrive at

$$
\begin{aligned}
& \mid E\left\{\exp \left(K\left(t_{n+1}-t_{k+1}\right)+c \int_{t_{k+1}}^{t_{n+1}}\left\|\nabla \omega\left(s^{\prime}, \cdot ; t_{k+1}, \omega\left(t_{k+1}, \cdot\right)\right)\right\|^{2} d s^{\prime}\right)\right. \\
& \left.\left(R\left(t_{k}, \cdot\right), \delta_{\omega}\left(t_{k+1}, \cdot\right)\right) \mid \mathcal{F}_{t_{k}}\right\} \mid \\
& =\mid\left(R\left(t_{k}, \cdot\right), E\left\{\exp \left(K\left(t_{n+1}-t_{k+1}\right)+c \int_{t_{k+1}}^{t_{n+1}}\left\|\nabla \omega\left(s^{\prime}, \cdot ; t_{k+1}, \omega\left(t_{k+1}, \cdot\right)\right)\right\|^{2} d s^{\prime}\right)\right.\right. \\
& \left.\left.\delta_{\omega}\left(t_{k+1}, \cdot\right) \mid \mathcal{F}_{t_{k}}\right\}\right) \mid \\
& \leq\left\|R\left(t_{k}, \cdot\right)\right\| \| E\left\{\exp \left(K\left(t_{n+1}-t_{k+1}\right)+c \int_{t_{k+1}}^{t_{n+1}}\left\|\nabla \omega\left(s^{\prime}, \cdot ; t_{k+1}, \omega\left(t_{k+1}, \cdot\right)\right)\right\|^{2} d s^{\prime}\right)\right. \\
& \left.\delta_{\omega}\left(t_{k+1}, \cdot\right) \mid \mathcal{F}_{t_{k}}\right\} \| \\
& =\left\|R\left(t_{k}, \cdot\right)\right\| \| E\left\{\left(E\left[\exp \left(K\left(t_{n+1}-t_{k+1}\right)+c \int_{t_{k+1}}^{t_{n+1}}\left\|\nabla \omega\left(s^{\prime}, \cdot ; t_{k+1}, \omega\left(t_{k+1}, \cdot\right)\right)\right\|^{2} d s^{\prime}\right)\right]\right.\right. \\
& \left.\left.+\sum_{r=1}^{q} \int_{0}^{t_{k+1}} \lambda_{r}(s) d w_{r}\right) \delta_{\omega}\left(t_{k+1}, \cdot\right) \mid \mathcal{F}_{t_{k}}\right\} \| \\
& \leq\left\|R\left(t_{k}, \cdot\right)\right\| E\left[\exp \left(K\left(t_{n+1}-t_{k+1}\right)+c \int_{t_{k+1}}^{t_{n+1}}\left\|\nabla \omega\left(s^{\prime}, \cdot ; t_{k+1}, \omega\left(t_{k+1}, \cdot\right)\right)\right\|^{2} d s^{\prime}\right)\right] \\
& \times\left\|E\left\{\delta_{\omega}\left(t_{k+1}, \cdot\right) \mid \mathcal{F}_{t_{k}}\right\}\right\|+\left\|R\left(t_{k}, \cdot\right)\right\|\left\|E\left\{\delta_{\omega}\left(t_{k+1}, \cdot\right) \sum_{r=1}^{q} \int_{0}^{t_{k+1}} \lambda_{r}(s) d w_{r} \mid \mathcal{F}_{t_{k}}\right\}\right\| \\
& \leq\left\|R\left(t_{k}, \cdot\right)\right\| C\left(t_{k}, \omega\right) h^{2}+\left\|R\left(t_{k}, \cdot\right)\right\|\left\|\sum_{r=1}^{q} \int_{0}^{t_{k}} \lambda_{r}(s) d w_{r} E\left\{\delta_{\omega}\left(t_{k+1}, \cdot\right) \mid \mathcal{F}_{t_{k}}\right\}\right\| \\
& +\left\|R\left(t_{k}, \cdot\right)\right\|\left(E\left[\left\|\delta_{\omega}\left(t_{k+1}, \cdot\right)\right\|^{2} \mid \mathcal{F}_{t_{k}}\right]\right)^{\frac{1}{2}}\left(\sum_{r=1}^{q}\left(E\left[\left\{\int_{t_{k}}^{t_{k+1}} \lambda_{r}(s) d w_{r}\right\}^{2} \mid \mathcal{F}_{t_{k}}\right]\right)^{\frac{1}{2}}\right) \\
& \leq\left\|R\left(t_{k}, \cdot\right)\right\| C\left(t_{k}, \omega\right) h^{2} \leq h\left\|R\left(t_{k}, \cdot\right)\right\|^{2}+\frac{h^{3}}{4} C^{2}\left(t_{k}, \omega\right) .
\end{aligned}
$$

Therefore,

$$
\begin{aligned}
& E\left\{\left(F_{k}-F_{k-1}\right) \exp \left(K\left(t_{n+1}-t_{k+1}\right)+c \int_{t_{k+1}}^{t_{n+1}}\left\|\nabla \omega\left(s^{\prime}, \cdot ; t_{k+1}, \omega\left(t_{k+1}, \cdot\right)\right)\right\|^{2} d s^{\prime}\right)\right\} \\
\leq & h E\left\|R\left(t_{k}, \cdot\right)\right\|^{2}+K h^{3} .
\end{aligned}
$$


From (5.9), (5.10), and (5.13), we obtain

$$
E\left\|R\left(t_{n+1}, \cdot\right)\right\|^{2} \leq h \sum_{k=1}^{n} E\left\|R\left(t_{k}, \cdot\right)\right\|^{2}+K h^{2},
$$

from which it follows by a version of Gronwall's lemma that

$$
E\left\|R\left(t_{n+1}, \cdot\right)\right\|^{2} \leq K h^{2},
$$

as required. Theorem 5.1 is proved.

The following corollary to Theorem 5.1 can be proved using the same approach as used in the proof of Corollary 3.1.

Corollary 5.1. Under the conditions of Theorem 5.1, the global approximation $\hat{v}_{k}(x):=$ $U \tilde{\omega}_{k}\left(t_{k}, x\right)$ of the velocity $v\left(t_{k}, x\right)$ has the first mean-square order accuracy, i.e., for a sufficiently small $h>0$ :

$$
\left(E\left\|v\left(t_{k}, \cdot\right)-\hat{v}_{k}(\cdot)\right\|^{2}\right)^{\frac{1}{2}} \leq K h, \quad k=1, \ldots, N,
$$

where $K>0$ is a constant independent of $k$ and $h$.

Remark 5.1. As we mentioned in the introduction, in this paper we aimed to prove the highest possible mean-square order of convergence (i.e., the first order in time step) for the proposed method from Section 4 under strong regularity assumptions on the solution: Theorem 5.1 is proved for the case when the SNSE solution $v(t, \cdot)$ of (2.1)(2.4) belongs to $\mathbf{V}_{p}^{5}$ (i.e., $m=3$ in Assumption 2.1), and consequently the vorticity $\omega(t, \cdot) \in \mathbf{H}_{p}^{4}$. In other papers (see, e.g., $[3,6]$ ), one typically establishes the convergence of methods under some prescribed, lower regularity conditions. Convergence of the method proposed in this paper under a lower regularity assumption is of interest and it can be a topic of future work. At the same time, let us observe the following. The higher regularity ( $m=3$ ) was needed to prove (2.39) of Lemma 2.2 and $(m=2)$ to prove Theorem 3.1 on the one-step error. It is possible to derive weaker versions of (2.39) and of Theorem 3.1 under $m=1$ (i.e., $\omega(t, \cdot) \in \mathbf{H}_{p}^{2}$ ), based on which an analogue of Theorem 5.1 giving just mean-square order $\frac{1}{4}$ can be proved. We also recall that existence of variational solutions of (2.1)-(2.4) requires $m=-1$ in Assumption 2.1 (i.e., $v(t, \cdot) \in \mathbf{V}_{p}^{1}$ ), while the vorticity formulation (2.19)-(2.21) requires $m=0$ (i.e., $\omega(t, \cdot) \in \mathbf{H}_{p}^{1}$ ). The benefit of the vorticity-velocity formulation is in avoidance of dealing with the divergence free condition imposed on the velocity $v$, with the price for that being the requirement for higher smoothness of the coefficients and the initial condition.

Remark 5.2. Various approaches can be used to turn the method, introduced at the start of Section 4 for the problem (2.19)-(2.21), into a numerical algorithm. To obtain a constructive numerical algorithm, we need to approximate the linear SPDE (3.2)(3.3) at every step in the mean-square sense, which is a much simpler problem than 
solving the SNSE (2.19)-(2.21). To this end, for instance, we can discretize this SPDE in space using the spectral method based on the Fourier expansion and use a finite difference for time discretization (see such an algorithm in the deterministic setting in e.g. [27]). Alternatively, we can apply the method based on averaging characteristics to (3.2)-(3.3) [24]. It is not difficult to construct such fully discrete algorithms, and we leave their analysis and testing for a future work.

Remark 5.3. In the preprint [26] we also considered deterministic (both two-dimensional and three-dimensional) incompressible NSE in the vorticity-velocity formulation with periodic boundary conditions, for which we proposed an approximation of vorticity similarly to the one constructed in this paper for SNSE. To compute the velocity, we derived a periodic version of Biot-Savart's law in the three dimensional case, which is analogous to the result for two-dimensional flows as e.g. in [15, pp. 50,71] and also (2.18) here. Further, in the deterministic case we suggested to use probabilistic representations together with ideas of weak-sense numerical integration of SDEs for solving the system of linear parabolic PDEs arising at every step of the vorticity approximation. We derived probabilistic representations appropriate for this task, which are based on [20] (see also [22,23]), and in the three-dimensional case these NSE-related representations are new.

\section{References}

[1] P. R. BEESACK, More generalised discrete Gronwall inequalities, ZAMM Z. Angew. Math. Mech., 65 (1985), 589-595.

[2] H. Bessaih, Z. BRZé́niaK, A. Millet, Splitting up method for the 2D stochastic NavierStokes equations, SPDEs: An. Comp., 2 (2014), 433-470.

[3] H. Bessain, A. Millet, Strong $L^{2}$ convergence of time numerical schemes for the stochastic two-dimensional Navier-Stokes equations, IMA J. Num. Anal., 39 (2019), 2135-2167.

[4] D. Breit, A. Dodgson, Convergence rates for the numerical approximation of the $2 D$ stochastic Navier-Stokes equations, arXiv:1906.11778.

[5] Z. BrzeźniaK, E. Carelli, A. Prohl, Finite element based discretizations of the incompressible Navier-Stokes equations with multiplicative random forcing, IMA J. Num. Anal., 34 (2014), 502-549.

[6] E. CARELLI, A. Prohl, Rates of convergence for discretizations of the stochastic incompressible Navier-Stokes equations, SIAM J. Num. Anal., 50 (2012), 2467-2496.

[7] P. Constantin, C. Foias, Navier-Stokes Equations, University of Chicago Press, 1988.

[8] P. DÖRSEK, Semigroup splitting and cubature approximations for the stochastic NavierStokes equations, SIAM J. Num. Anal., 50 (2012), 729-746.

[9] Y. DUAN, X. YANG, The finite element method of a Euler scheme for stochastic Navier-Stokes equations involving the turbulent component, Int. J. Numer. Anal. Mod., 10 (2013), 727744.

[10] F. FLANDOLI, An introduction to 3D stochastic fluid dynamics. In: SPDE in Hydrodynamic: Recent Progress and Prospects, Lecture Notes in Mathematics, 1942, Springer, 2008, 51150.

[11] C. Foias, O. Manley, R. Rosa, R. Temam, Navier-Stokes Equations and Turbulence, Cambridge University Press, 2001. 
[12] V. Girault, P. A. RaviarT, Finite Element Methods for Navier-Stokes Equations, Springer, 1986.

[13] M. Hairer, J. C. MatTingly, Ergodicity of the 2D Navier-Stokes equations with degenerate stochastic forcing, Ann. Math., 164 (2006), 993-1032.

[14] R. Kruse, M. Scheutzow, A discrete stochastic Gronwall lemma, Math. Comp. Simul., 143 (2018), 149-157.

[15] A. J. MAJdA, A. L. Bertozzi, Vorticity and Incompressible Flow, Cambridge Univ. Press, 2003.

[16] J. C. Mattingly, The Stochastic Navier-Stokes Equation: Energy Estimates and Phase Space Contraction, Ph.D. Thesis, Princeton University, 1998.

[17] J. C. Mattingly, The dissipative scale of the stochastics Navier-Stokes equation: regularization and analyticity, J. Stat. Phys., 108 (2002), 1157-1179.

[18] J. C. MatTingly, E. PARDoux, Malliavin calculus and the randomly forced Navier-Stokes equation, Comm. Pure Appl. Math., 59 (2006), 1742-1790.

[19] R. Mikulevicius, B. RozovskiI, Global L2-solutions of stochastic Navier-Stokes equations, Ann. Prob., 33 (2005), 137-176.

[20] G. N. Milstein, Probabilistic solution of linear systems of elliptic and parabolic equations, Theor. Prob. Appl., 23 (1978), 851-855.

[21] G. N. Milstein, A theorem on the order of convergence of mean-square approximations of solutions of systems of stochastic differential equations, Teor. Veroyat. Primenen., 32 (1987), 809-811.

[22] G. N. Milstein, J. G. M. Schoenmakers, V. Spokoiny, Transition density estimation for stochastic differential equations via forward-reverse representations, Bernoulli, 10 (2004), 281-312.

[23] G. N. Milstein, M. V. Tretyakov, Stochastic Numerics for Mathematical Physics, Springer, 2004.

[24] G. N. Milstein, M. V. TRetyakov, Solving parabolic stochastic partial differential equations via averaging over characteristics, Math. Comp., 78 (2009), 2075-2106.

[25] G. N. Milstein, M. V. TRetyakov, Layer methods for Navier-Stokes equations with additive noise using simplest characteristics, J. Comp Appl. Maths., 302 (2016), 1-23.

[26] G. N. Milstein, M. V. TRetyakov, Approximation of deterministic and stochastic NavierStokes equations in vorticity-velocity formulation, Preprint. arXiv:1804.07289.

[27] R. Peyret, Spectral Methods for Incompressible Viscous Flow, Springer, 2002.

[28] R. Temam, Navier-Stokes Equations and Nonlinear Functional Analysis, SIAM, 1995.

[29] P. Wesseling, Principles of Computational Fluid Dynamics, Springer, 2001. 